\title{
Isoleucine 44 Hydrophobic Patch Controls Toxicity of Unanchored, Linear Ubiquitin Chains through NF-кB Signaling
}

\author{
Jessica R. Blount ${ }^{1}$, Kozeta Libohova ${ }^{1}$, Gustavo M. Silva ${ }^{2}$ and Sokol V. Todi ${ }^{1,3, *(D)}$ \\ 1 Department of Pharmacology, Wayne State University School of Medicine, 540 East Canfield St., \\ Scott Hall Rm. 3108, Detroit, MI 48201, USA; jrblount@wayne.edu (J.R.B.); klibohov@med.wayne.edu (K.L.) \\ 2 Department of Biology, Duke University, Durham, NC 27708, USA; gustavo.silva@duke.edu \\ 3 Department of Neurology, Wayne State University School of Medicine, 540 East Canfield St., \\ Scott Hall Rm. 3108, Detroit, MI 48201, USA \\ * Correspondence: stodi@wayne.edu
}

Received: 7 April 2020; Accepted: 16 June 2020; Published: 22 June 2020

\begin{abstract}
Ubiquitination is a post-translational modification that regulates cellular processes by altering the interactions of proteins to which ubiquitin, a small protein adduct, is conjugated. Ubiquitination yields various products, including mono- and poly-ubiquitinated substrates, as well as unanchored poly-ubiquitin chains whose accumulation is considered toxic. We previously showed that transgenic, unanchored poly-ubiquitin is not problematic in Drosophila melanogaster. In the fruit fly, free chains exist in various lengths and topologies and are degraded by the proteasome; they are also conjugated onto other proteins as one unit, eliminating them from the free ubiquitin chain pool. Here, to further explore the notion of unanchored chain toxicity, we examined when free poly-ubiquitin might become problematic. We found that unanchored chains can be highly toxic if they resemble linear poly-ubiquitin that cannot be modified into other topologies. These species upregulate NF- $\mathrm{kB}$ signaling, and modulation of the levels of NF- $\mathrm{kB}$ components reduces toxicity. In additional studies, we show that toxicity from untethered, linear chains is regulated by isoleucine 44 , which anchors a key interaction site for ubiquitin. We conclude that free ubiquitin chains can be toxic, but only in uncommon circumstances, such as when the ability of cells to modify and regulate them is markedly restricted.
\end{abstract}

Keywords: Drosophila melanogaster; innate immunity; NF-кB signaling; ubiquitination; unanchored poly-ubiquitin

\section{Introduction}

Post-translational modification adds a critical layer of complexity to the genome, allowing for regulation of many cellular functions. Methylation of histones in nucleosomes controls the expression of specific genes [1], phosphorylation of enzymes determines their activity [2], and glycosylation affects a protein's conformation and ability to form complexes [3]. Ubiquitin ( $\mathrm{Ub})$ is a small protein adduct that is arguably known best for its role in protein quality control due to its participation in proteasome-dependent degradation [4]. Beyond that, $\mathrm{Ub}$ is an essential player in nearly all cellular processes, making it vital to eukaryotic cell health. A protein's ubiquitination status can lead to various outcomes, from alteration of its protein-protein interactions to changes in its localization, function, enzymatic activity and half-life [5-9].

Ubiquitination is a sequential, ATP-dependent process that requires a Ub-activating enzyme (E1), a Ub-conjugating enzyme (E2), and a Ub ligase (E3) to covalently attach a Ub molecule to a substrate protein, typically at one of the substrate's lysine residues [5]. With seven lysine residues of its own, 
$\mathrm{Ub}$ itself becomes ubiquitinated, resulting in the formation of poly-Ub chains with unique topologies dependent on the specific lysine residue linkers. Head-to-tail chains also exist, linked at the N-terminal methionine, as well as chains that contain a mixture of linkage types $[10,11]$. Ub chains that are not attached to a protein-known as unanchored, untethered, or free poly- $\mathrm{Ub}$ - can be synthesized by specialized E2s and E3s, and also arise from the removal of a preexisting chain from a ubiquitinated protein by deubiquitinating enzymes (DUBs) [12].

Ubiquitination is editable and reversible, ensuring that ubiquitinated substrates are targeted to the proper cellular complex while maintaining an available pool of $\mathrm{Ub}$ for reutilization. DUBs are a family of enzymes responsible for cleaving the linkages of $\mathrm{Ub}$ with its substrate or the linkages between $\mathrm{Ub}$ monomers in a poly-Ub chain. Depending on the DUB, it may influence the outcome for a ubiquitinated protein by editing the length or topological makeup of the $\mathrm{Ub}$ chain, or by removing the chain entirely [12-14]. In the case of unanchored poly-Ub, specific DUBs break down multiple, linked $\mathrm{Ub}$ molecules into mono-Ub; such is the case with Ubiquitin-Specific Protease 5, which has been evidenced to disassemble unanchored chains [15]. Processing of unanchored poly-Ub is important, as these chains participate in several processes, such as NF-kB signaling, and because disassembly of unanchored poly- $\mathrm{Ub}$ can replenish the pool of mono- $\mathrm{Ub}$ for reuse [14,16-20].

Unanchored poly-Ub has not been as well studied as other $\mathrm{Ub}$ species. There are several concepts regarding free poly-Ub based on studies in yeast and cultured mammalian cells: that it exists only at very low concentrations, that it is quickly disassembled by DUBs to be reused as mono-Ub, and that its accumulation is toxic $[9,12,14,21,22]$. It has been proposed that unanchored poly-Ub interferes with the normal function of the proteasome, either by directly interacting with and occluding it-i.e., free poly-Ub could begin its own degradation process, but overwhelms the proteasomal machinery-or by outcompeting ubiquitinated proteasomal substrates [23-25].

Our previous work introduced transgenic, DUB-resistant, untethered Ub chains into a physiological system, Drosophila melanogaster, to address questions surrounding the potential toxicity and handling of free poly- $\mathrm{Ub}$ in an intact, multicellular organism [26,27]. We synthesized DNA to express six Ub molecules in tandem, without any internal "GG" motifs (denoted as $\mathrm{Ub}^{6}$ ), which prevents them from being cleaved into mono-Ub by DUBs. $\mathrm{Ub}^{6}$ chains exhibited little-to-no toxicity, did not interfere with proteasomal function, and were readily subjected to proteasomal degradation [26]. These chains could also be transferred to a substrate protein en bloc in flies and in mammalian cells, without the need for prior disassembly into mono-Ub [26]. The DUB-resistant, free $\mathrm{Ub}^{6}$ chains become ubiquitinated themselves through various linkages, transforming them into mixed-linkage, free chains [26]. Additional work indicated that the continuous presence of these $\mathrm{Ub}$ chains in vivo does not cause marked changes at the transcriptome level in the fly [27]. Collectively, we interpreted these results to indicate that free poly- $\mathrm{Ub}$ is not necessarily detrimental in vivo and that $\mathrm{Ub}$ recycling may take place not only at the level of the monomer, but also through the reutilization of intact, untethered $\mathrm{Ub}$ chains.

Nonetheless, considering earlier studies that suggested toxicity from free chains $[23,24,28]$, we sought to understand under what circumstances unanchored poly-Ub might become problematic to the cell. One key feature of the transgenic $\mathrm{Ub}$ chains expressed in flies is their conjugation with endogenous $\mathrm{Ub}$ [26]. This phenomenon made us wonder whether ubiquitination of these chains is important for the lack of toxicity we observe. Therefore, we generated new, isogenic fly lines that express $\mathrm{Ub}^{6}$ identical to the previously described species, with the exception that they lack all internal lysine residues, rendering them ubiquitination-resistant. The new chains essentially resemble free, linear poly-Ub. By manipulating the potential of $\mathrm{Ub}^{6}$ to be ubiquitinated, rather than the cell's ability to ubiquitinate proteins, we avoid impacting ubiquitination processes more generally and can attribute changes in toxicity to the presence of $\mathrm{Ub}^{6}$.

We found stark differences in the toxicity of and response to ubiquitination-resistant $\mathrm{Ub}^{6}$ chains in vivo. Depending on the type of tissue where lysine-less $\mathrm{Ub}^{6}$ was expressed, it led to either developmental lethality or markedly shortened adult lifespan. Ubiquitination-resistant $\mathrm{Ub}^{6}$ has an 
extended turnover cycle when compared to $\mathrm{Ub}^{6}$ with all its lysine residues intact. Additionally, ubiquitination-resistant $\mathrm{Ub}^{6}$ leads to the upregulation of several components of NF- $\mathrm{kB}$ pathways in the fly, indicating a mechanism for toxicity from these free chains in Drosophila. Importantly, toxicity from ubiquitination-resistant $\mathrm{Ub}^{6}$ is reversed by mutating isoleucine (ile) residues at position 44 of each constituent $\mathrm{Ub}$ into alanine. Ile44 is a key part of a hydrophobic patch on $\mathrm{Ub}$ that controls many interactions [5,29-32]. We conclude that while unanchored poly-Ub is not inherently problematic in vivo, there can be circumstances in which these species may become detrimental, such as when cells' abilities to manipulate and handle them are severely hampered. The studies that we describe here also introduce to the field new genetic tools for continued investigation of untethered poly-Ub.

\section{Materials and Methods}

\subsection{Antibodies}

Anti-HA (1:500 or 1:1000; rabbit monoclonal; Cell Signaling Technology, Danvers, MA, USA, \#3724); anti-Ub (1:1000; rabbit polyclonal; Cell Signaling Technology, \#3933); anti-tubulin (1:1000, mouse monoclonal, Sigma-Aldrich, St. Louis, MO, USA, \#T5168); anti-Relish (1:500 from concentrate; mouse monoclonal; Developmental Studies Hybridoma Bank, Iowa City, IA, USA, \#21F3); anti-lamin (1:1000; mouse monoclonal; Developmental Studies Hybridoma Bank, \#ADL84.12); anti-phosphorylated-p65 (serine 536; 1:1000; rabbit monoclonal; Cell Signaling Technology, \#3033); anti-p65 (1:1000; rabbit monoclonal; Cell Signaling Technology, \#8242); goat anti-mouse, peroxidase-conjugated secondary (1:10,000; Jackson Immunoresearch, West Grove, PA, USA); goat anti-rabbit, peroxidase-conjugated secondary (1:10,000; Jackson Immunoresearch).

\subsection{Fly Generation}

GenScript (genscript.com; Piscataway, NJ, USA) synthesized $\mathrm{Ub}^{6}$ transgenes and cloned them into pWalium10.moe by using restriction sites EcoRI and XbaI, as also described before [26]. The amino acid sequences of the new lines generated for this study are shown in Figure 1 and Figure 5. The purified plasmid was injected into yw; attP40 by Duke University Model Systems, using the PhiC31-targeted integration to achieve site-specific, unidirectional integration into the second chromosome [33,34]. The transformed chromosome was migrated onto the $\mathrm{w}^{1118}$ parental line and balanced lines were confirmed through genomic DNA-based PCR assays for transgene insertion and orientation, as described before $[35,36]$. All fly lines were sequence verified.

\subsection{Drosophila Stocks and Procedures}

All flies were maintained in diurnal incubators at $25{ }^{\circ} \mathrm{C}$ and $\sim 60 \%$ humidity, in conventional cornmeal media. Where noted, RU486 was added to the conventional media, as previously described [37]. Tubulin-Gal4-GS was a generous gift from Dr. R.J. Wessells, Wayne State University; sqh-Gal4 was a gift from Dr. Daniel Kiehart, Duke University; repo-Gal4 was gifted by Dr. Daniel Eberl, University of Iowa. Mef2-Gal4 (\#27390) and elav-Gal4 (\#458) were from Bloomington Drosophila Stock Center (Bloomington, IN, USA). The following RNAi lines were also from the Bloomington Drosophila Stock Center: Relish (\#3361), Dif (\#30513), cactus (\#31713), dorsal (\#27650), IKKBeta (\#35186), kenny (\#35572, 57759). dorsal overexpression line was from FlyORF (Zurich, Switzerland, \#000638). For longevity assays, male and female flies were collected as they eclosed from their pupal cases and aged on conventional cornmeal fly media at $25^{\circ} \mathrm{C}$, with $\sim 20$ flies per vial. Flies were transferred to fresh vials every 2-3 days until all were dead. Throughout this study, flies were heterozygous for driver and transgenes. 


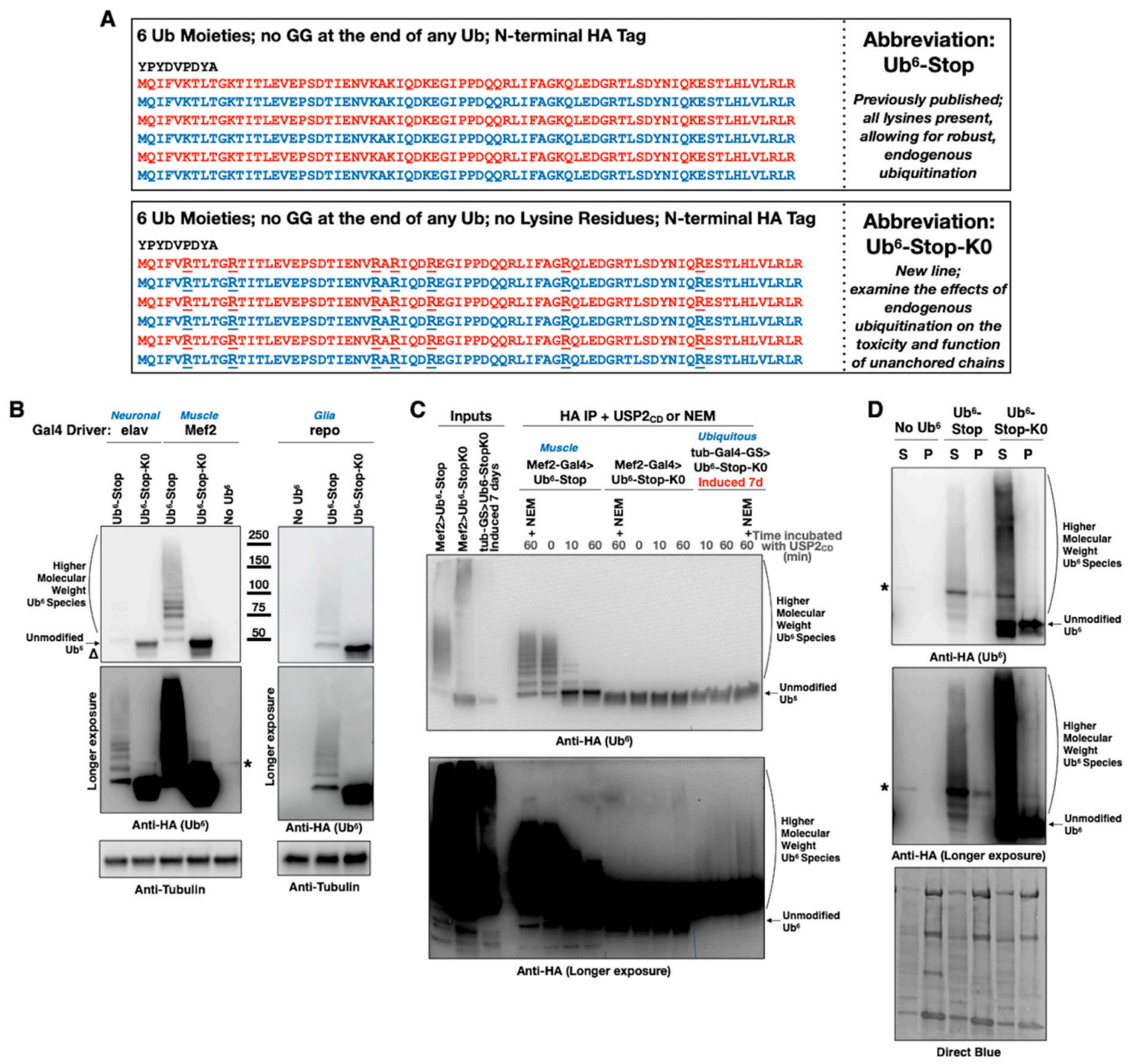

Figure 1. New transgenic Drosophila lines expressing non-cleavable, unanchored ubiquitin chains. (A) Amino acid sequence, abbreviation and purpose of HA-tagged ubiquitin chain transgenes. Neither chain can be conjugated onto another protein as it lacks a terminal "GG" motif that is required for isopeptide bond formation. Absence of internal "GG" motifs also ensures that they are not dismantled by DUBs [26]. All lysine (K) residues within $\mathrm{Ub}^{6}$-Stop-K0 were mutated into the similar, but non-ubiquitinatable, amino acid arginine (R) to prevent ubiquitination. (B) Western blots from flies expressing the noted transgenes in all neurons (elav-Gal4, adult lysates), muscle cells (Mef-Gal4, adult lysates), or all glial cells (repo-Gal4, pharate adult lysates). Delta symbol $(\Delta)$ ): signal underneath the main band of lysine-less $\mathrm{Ub}^{6}$ that we observe sometimes and could be a proteolytic fragment of the chain. (C) Western blots from stringently immunopurified, HA-tagged ubiquitin chains treated, or not, with the catalytic domain of USP2 for the indicated amounts of time. Mef2-Gal4 flies were one day old. Flies with tub-Gal4-GS driver were induced to express $\mathrm{Ub}^{6}$ for 7 days before being collected for protein extraction. (D) Western blots from soluble/pellet fractionation of flies expressing the noted ubiquitin chains in all muscle cells. Flies were one day old. The smear present in the Ub ${ }^{6}-\mathrm{Stop}-\mathrm{K} 0$ samples comprises SDS-resistant species as a result of the buffer used in this protocol. As shown in Figure $1 C$, second lane from the left and USP2 ${ }_{C D}$-treated lanes, similar smears from a different buffer and lysis protocol (Materials and Methods) are not collapsed by the addition of the DUB. Asterisks in panels: non-specific band detected by the anti-HA antibody. 


\subsection{Western Blotting}

Five whole adults flies or pharate adults, or ten dissected heads per group were lysed as described previously [26]. Blots were developed and quantified using a CCD-equipped VersaDoc system and Quantity One software (Bio-Rad, Hercules, CA, USA; version 4.6.8), Syngene PXi4 and GeneSys software (Syngene, Frederick, MD, USA; version 1.7.2), or ChemiDoc and ImageLab (Bio-Rad; version 6.0.1).

\subsection{Immunoprecipitation and In Vitro Deubiquitination}

For precipitation of HA-Ub ${ }^{6}$ from flies, 40 flies per group were homogenized in RIPA lysis buffer (50 mM Tris, $150 \mathrm{mM} \mathrm{NaCl}, 0.1 \%$ SDS, 0.5\% deoxycholic acid, 1\% NP40, pH 7.4) supplemented with protease inhibitor cocktail (PI; Sigma-Aldrich), sonicated, centrifuged at $15,000 \times g$ for 20 min at $4{ }^{\circ} \mathrm{C}$, and then incubated with anti-HA antibody-bound beads for four hours, tumbling at $4{ }^{\circ} \mathrm{C}$. Beads were rinsed 10 times (including $2 \times 5 \mathrm{~min}$ tumbling at $4{ }^{\circ} \mathrm{C}$ ), and then split into two microfuge tubes and incubated at $37^{\circ} \mathrm{C}$ with either NEM $(0.5 \mathrm{mM})$ or the catalytic domain of USP2 $(0.1 \mu \mathrm{M}$; Boston Biochem, Cambridge, MA, USA). After incubation for the selected times, reactions were stopped by the addition of $6 \%$ SDS sample buffer and by boiling for $1 \mathrm{~min}$.

\subsection{Soluble/Insoluble Fractionation}

Five flies per group were mechanically homogenized in $200 \mu \mathrm{L}$ of NETN lysis buffer ( $50 \mathrm{mM}$ Tris $\mathrm{pH} 7.5,150 \mathrm{mM} \mathrm{NaCl}$, and 0.5\% IPEGAL ca-630) supplemented with PI, sonicated briefly, and then centrifuged at $20,000 \times \mathrm{g}$ for $30 \mathrm{~min}$ at $4{ }^{\circ} \mathrm{C}$. The resulting supernatant was removed, transferred into a new microfuge tube, and subsequently quantified by using the BCA assay (ThermoFisher, Waltham, MA, USA). The remaining pellet was fully resuspended in $200 \mu \mathrm{L}$ of PBS $+1 \%$ SDS by vortexing and boiling. Then, $10 \mu \mathrm{g}$ of the supernatant fraction and $7 \mu \mathrm{L}$ of the pellet fraction were supplemented with loading buffer, boiled briefly and electrophoresed on SDS-PAGE gels for analyses by Western blotting.

\subsection{Quantitative Real-Time PCR}

From 10 dissected pharate heads per group, total RNA was extracted using TRIzol reagent (Invitrogen, Carlsbad, CA, USA) and treated with TURBO DNase (Ambion, Austin, TX, USA) to eliminate contaminating DNA. A high-Capacity cDNA Reverse Transcription Kit (ABI, Waltham, MA, USA) was used to obtain a cDNA library, and preamplification of the genes of interest was performed using SsoAdvanced Preamplification Supermix (Bio-Rad). A Fast SYBR Green Master Mix (ThermoFisher) was used in conjunction with a StepOne real-time PCR system. mRNA levels were quantified using $2^{-\Delta \Delta \mathrm{Ct}}$ (cycle threshold) methods and normalizing all transcripts to the reference gene, rp49. The following primer sequences were used:

rp49 (F: AGATCGTGAAGAAGCGCACCAAG; R: CACCAGGAACTTCTTGAATCCGG), Relish (F: ATGAACTTGAACCAGGTGCGG; R: TGCCGACTTGCGGTTATTGA), Dif (F: CAGAGTTCCAACCCACGGAC; R: AGGAGTTCTGGATTCGGGTAGT), dorsal (F: AGAGCCCGCCAAGGTTTTT; R: TGCCATCCTGGTGGTCATTC), cactus (F: ATCCAACGACAAAGCGGTCA; R: GATTTTCCCTCCCTGGCGTTA), and kenny (F: TACCTCGCGCTAAAGAGCAC; R: CAGCTCTTGGTTTTCCACGC).

\subsection{Subcellular Fractionation}

A ReadyPrep Cytoplasmic/Nuclear Protein Extraction Kit (Bio-Rad) was used to separate nuclear and cytoplasmic proteins from 10 pharate adult dissected heads, or individual wells in a 24-well culture plate for experiments with HEK-293T cells. The manufacturer's protocol was used, with two variations: (1) nuclear fractions were rinsed once with cytoplasmic protein exclusion buffer (CPEB) for $10 \mathrm{~min}$, tumbling at $4{ }^{\circ} \mathrm{C}$ before subsequent incubations in CPEB on ice; (2) cytoplasmic fractions were centrifuged $3 \times$, with the supernatant transferred to a fresh tube between spins. 


\subsection{Mammalian Cell Maintenance and Transfection}

HEK-293T cells were maintained under conventional conditions in DMEM with 10\% FBS and $5 \%$ Penicillin-Streptomycin. Cells were transfected using Lipofectamine LTX (Invitrogen), using the manufacturer's protocols. Twenty-four hours after transfection, cells were collected and rinsed in cold PBS, and then used for whole-cell lysis (using the same buffer and protocol as for blots with flies), or for subcellular fractionation. DNA constructs used for transfections were the same as those synthesized for fly generation, but subcloned into the mammalian vector pcDNA3.1.

\section{Results}

\subsection{New Drosophila Lines Expressing Ubiquitination-Resistant, Unanchored Chains}

As introduced above, we previously examined whether expression of unanchored poly-Ub is toxic in Drosophila. Using the Gal4-UAS system [38,39], we expressed $\mathrm{Ub}^{6}$ chains that cannot be dismantled by DUBs (e.g., $\mathrm{Ub}^{6}$-Stop in Figure 1A). These poly-Ub resemble linear, untethered chains that exist in nature $[5,14,21]$. The chains were constructed to either remain unanchored, due to the lack of the terminal "GG" motif that would enable their isopeptide-bond fusion to another protein $\left(\mathrm{Ub}^{6}\right.$-Stop; Figure 1A), or could be tethered wholesale onto another protein as a result of a single "GG" motif at the very end [26]. We found that these $\mathrm{Ub}^{6}$ species are innocuous [26,27]. Nonetheless, as some studies concluded that untethered poly-Ub is toxic $[23,24,28]$, we sought to explore when free chains might become detrimental to the cell. We focused on a potential role from the ubiquitination of untethered poly-Ub by endogenous $\mathrm{Ub}$, since this is a major feature of transgenically-expressed chains [26].

We designed head-to-tail $\mathrm{Ub}^{6}$ that, similar to the prior constructs [26], does not contain "GG" motifs internally or at the very end, and is also devoid of lysine residues (lys to arg mutation), making it resistant to ubiquitination ( $\mathrm{Ub}^{6}$-Stop-K0; Figure $\left.1 \mathrm{~A}\right)$. Removing the potential for ubiquitination of $\mathrm{Ub}^{6}$ allows us to examine the behavior of an untethered $\mathrm{Ub}$ chain that cannot be decorated by ubiquitination within the cell, in contrast to the mixed-linkage chains that arose and were highly abundant in our previous studies [26].

We generated new transgenic flies that incorporate $\mathrm{Ub}^{6}-\mathrm{Stop}-\mathrm{K} 0$ at the same chromosomal site that contains $\mathrm{Ub}^{6}$-Stop, attP40 on the second chromosome of Drosophila. The PhiC31 system of transgene integration ensures single copy, same orientation insertion of the intended construct into the same chromosomal site [26,33,34,40-42]. Following established protocols from our published work [35,36], we utilized PCR-based assays and sequencing to ensure integration of the transgene into the right locus and in the correct orientation, and to ensure that the sequence inserted was the one intended (data not shown). With these isogenic lines on hand, we began our investigations. To characterize the biochemical properties of $\mathrm{Ub}^{6}$-Stop-K0 in comparison to $\mathrm{Ub}^{6}$-Stop, we first performed Western blotting using lysates collected from flies expressing either version of $\mathrm{Ub}^{6}$. We observed $\mathrm{Ub}^{6}$-Stop-K0 at the expected molecular weight of $\sim 50 \mathrm{kDa}$, without the distinct and clearly defined ubiquitination laddering that we see above $\mathrm{Ub}^{6}$-Stop (Figure $1 \mathrm{~B}$ and other supportive information in data collected for Sections 3.3 and 3.5). At this point, it is interesting to note that the precise type of ubiquitination laddering above the main band of $\mathrm{Ub}^{6}$-Stop differs among different tissues. In muscle cells, there appears to be a higher preponderance of $>\mathrm{Ub}^{1}$ addition $\left(\mathrm{Ub}^{3-5}\right.$, middle portion of the blots in Figure 1B; see also data pertaining to Section 3.5.) compared to neuronal and glial cells, whose signal is stronger closer to the main, unmodified band. The reasons for these differences and their potential physiological consequences are unknown. Still, the key point from these comparisons is that $\mathrm{Ub}^{6}$-Stop-K0 migrates similarly in different fly tissues.

We showed previously that the laddering above the main band of $\mathrm{Ub}^{6}$-Stop comprises ubiquitinated species [26]. One of the methods we utilized was to incubate immunoprecipitated (IP-ed) Ub 6 -Stop with the catalytic domain of USP2 (USP2 $C D$ ), a DUB that would remove $\mathrm{Ub}$ added onto $\mathrm{Ub}^{6}$ in any type of linkage, including met1/linear fusions [43], while leaving $\mathrm{Ub}^{6}$ itself intact due to absence of "GG" motifs. As shown in Figure 1C, while USP2 ${ }_{C D}$ readily removes conjugated $\mathrm{Ub}$ from $\mathrm{Ub}^{6}$-Stop, 
as evidenced by the collapse of the higher molecular weight (HMW) bands into the main $\mathrm{Ub}^{6}$ band, it has no detectable effect on $\mathrm{Ub}^{6}$-Stop-K0. The faint HMW smear observed in the long-exposure image in Figure $1 \mathrm{C}$ is likely aggregated $\mathrm{Ub}^{6}$-Stop-K0, as suggested by centrifugation protocols (Figure 1D). Through centrifugation of fly lysates, we observe the presence of some $\mathrm{Ub}^{6}$-Stop, and especially of $\mathrm{Ub}^{6}$-Stop-K0, in the pellet fraction, suggesting that both chains may aggregate (Figure 1D). Together, these data indicate that $\mathrm{Ub}^{6}$-Stop-K0 is expressed in the fly and that it is resistant to ubiquitination.

\subsection{Lysine-Less, Unanchored Poly-Ub Is Highly Toxic in Drosophila}

We found before that expression of $\mathrm{Ub}^{6}$-Stop in all tissues or only in muscle cells did not impact longevity, while restricted expression in glial cells or neurons reduced adult fly longevity slightly, by a few days (normal flies can live for approximately 90 days; [26]). Here, we used various Gal4-UAS drivers to test the impact of $\mathrm{Ub}^{6}$-Stop and $\mathrm{Ub}^{6}$-Stop-K0 in fly longevity. sqh-Gal4 is a commonly used driver that leads to expression of the transgene of interest in all tissues during development and as adults $[26,40,41,44,45]$. Flies heterozygous for sqh-Gal4 and $\mathrm{Ub}^{6}$-Stop-K0 die as embryos or young larvae (Figure 2A). elav-Gal4 (pan-neuronal expression) and repo-Gal4 (pan-glial expression) both lead to developmental death and drastically shortened adult lifespans when expressing $\mathrm{Ub}^{6}$-Stop-K0 (Figure 2A-C). In the case of glial expression, no $\mathrm{Ub}^{6}$-Stop-K0-expressing adults emerged from most crosses that we set up; developing flies died as pharate adults. We were able to collect some live adults in later crosses for experiments described in Section 3.4 and their lives were significantly shorter than control adults. It is the longevity of these adults that is shown in Figure 2C. Expression of $\mathrm{Ub}^{6}$-Stop-K0 in muscle cells (Mef2-Gal4) results in a less dramatically, but still statistically significantly abbreviated lifespan (Figure 2A,D). In essence, with every Gal4 driver used, Ub ${ }^{6}$-Stop-K0 leads to markedly reduced lifespan compared to both $\mathrm{Ub}^{6}$-Stop flies and control flies that are genetically similar but do not express either $\mathrm{Ub}^{6}$ (Figure 2). Clearly, different drivers lead to variable toxicity from ubiquitination-resistant, linear poly-Ub. Among the tissues examined, muscle cells are least impacted. This is unlikely to be directly due to differences in relative amounts expressed by the different drivers, since we showed before that muscle cells express $\mathrm{Ub}^{6}$ transgenes highly robustly [26]. More likely, different tissues handle and tolerate these species in different ways, leading to the observed variation in toxicity. These findings highlight the need to investigate $\mathrm{Ub}$-dependent pathways in a tissue-specific manner to understand the full scope of their roles and importance in vivo. Collectively, these data point to marked toxicity from ubiquitination-resistant, untethered $\mathrm{Ub}^{6}$ species in Drosophila, unlike the ubiquitination-prone counterpart.

\subsection{Lysine-Less, Unanchored Poly-Ub Is More Stable in the Fly}

Since endogenous ubiquitination may influence the toxicity of $\mathrm{Ub}^{6}$, we questioned whether fly cells clear $\mathrm{Ub}^{6}$-Stop-K0 as readily as $\mathrm{Ub}^{6}$-Stop, which can be ubiquitinated. Lys48- and lys11-linked ubiquitination are proteasomal degradation signals $[4,5,46,47]$ that are present on $\mathrm{Ub}^{6}$-Stop ([26] and unpublished observations from J. R. Blount and S. V. Todi). It is not unreasonable to hypothesize that their absence could hinder the degradation of $\mathrm{Ub}^{6}$-Stop-K0. To determine whether $\mathrm{Ub}^{6}-\mathrm{S}$ top-K0 is turned over more slowly than $\mathrm{Ub}^{6}$-Stop, we employed the RU486-dependent, ubiquitous driver, tub-Gal4-GS to perform an experiment akin to a pulse-chase [26]. Flies heterozygous for tub-Gal4-GS and either form of $\mathrm{Ub}^{6}$ were reared on regular fly food until they emerged from their pupal cases, at which point they were switched to RU486 food for 2 days to induce $\mathrm{Ub}^{6}$ transgene expression (the "pulse"). Adults were then switched to regular media to halt additional $\mathrm{Ub}^{6}$ production and were flash-frozen periodically to assess $\mathrm{Ub}^{6}$ disappearance by Western blotting (the "chase"). We selected an inducible, ubiquitous Gal4 driver and focused on adult flies to gain a general perspective on the turnover of these $\mathrm{Ub}$ species, aware of the fact that degradation rates may differ in a tissue- and developmental stage-dependent manner. Nonetheless, this approach provides us with critical insight into the handling of ubiquitination-prone and ubiquitination-resistant, linear poly- $\mathrm{Ub}$ at the level of the whole organism. 
A

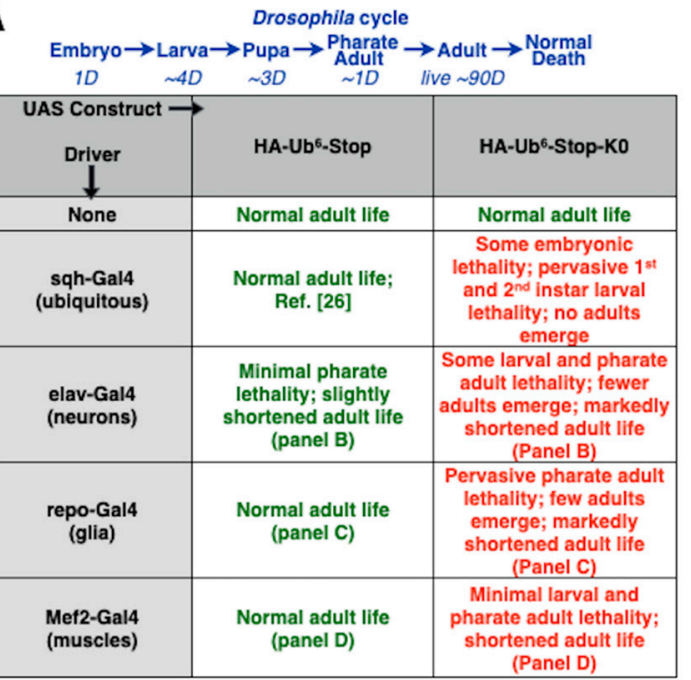

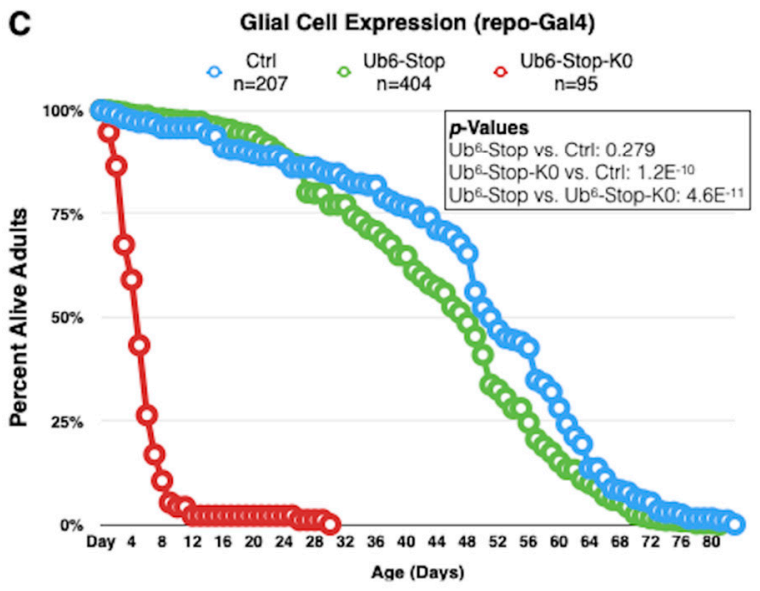

B

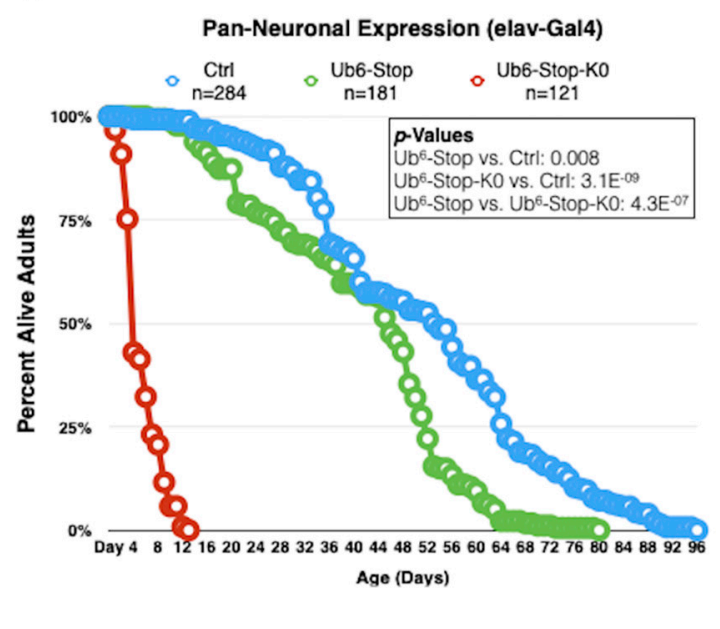

D

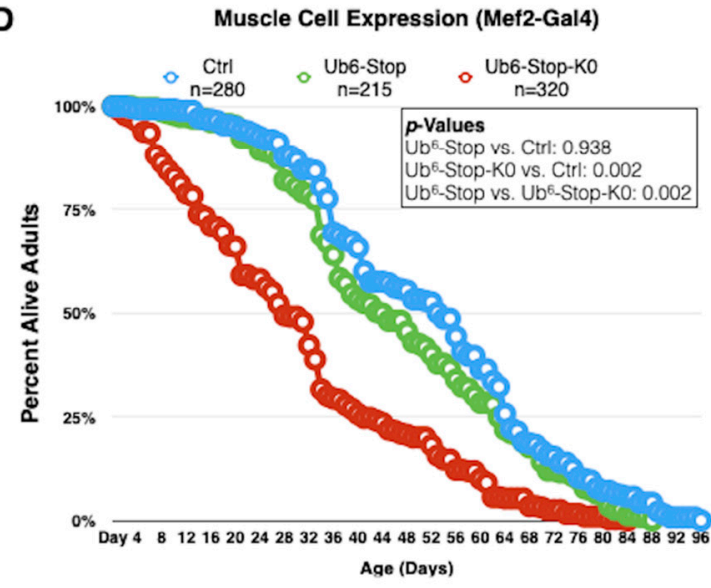

Figure 2. Lysine-less, non-cleavable ubiquitin chains are toxic in flies. (A) Summary of findings when the noted transgenes are expressed as indicated. "None" denotes flies that contained the indicated transgene without a Gal4 driver. Results are from crosses that were conducted simultaneously. A schematic of the fly life cycle, with the approximate number of days ("D") spent in each stage, is provided. (B-D) Longevity assays of adult flies expressing $\mathrm{Ub}^{6}$ chains under the noted Gal4 drivers. "Ctrls" do not contain $\mathrm{Ub}^{6}$ transgenes, but have the Gal4 driver on the genetic background that we utilized to generate the ubiquitin chain-containing flies. ' $p$ ' values are from log-rank tests.

Figure 3 summarizes our findings. We observe that the lack of ubiquitination stabilizes $\mathrm{Ub}^{6}-\mathrm{Stop}-\mathrm{K} 0$ compared to $\mathrm{Ub}^{6}$-Stop. Fifty percent of $\mathrm{Ub}^{6}-\mathrm{Stop}-\mathrm{K} 0$ is degraded by approximately $72 \mathrm{~h}$ in comparison to less than $24 \mathrm{~h}$ for $\mathrm{Ub}^{6}$-Stop. By 7 days, both proteins reach comparable levels. These data suggest that internal lysine residues and their ubiquitination are critical, but not entirely essential for chain turnover in flies.

\subsection{Toxicity of Lysine-Less, Unanchored Poly-Ub Depends on NF- $\kappa B$ Signaling}

Recent studies focusing on innate immunity pathways have revealed roles for free poly-Ub in NF- $\kappa$ B signaling cascades [16,48-52]. "NF- $\kappa B$ " refers to a family of inducible transcription factors which, in response to the activation of immune receptors by invading microbes, induce the expression of antimicrobial peptides. In Drosophila, innate immunity is primarily regulated by two pathways that activate NF- $\kappa$ B: Imd and Toll (Figure 4A). During the Drosophila immune response mediated by NF- $\kappa$ B pathways, a series of downstream regulators are involved (e.g., cactus, dorsal, kenny, and Relish; Figure $4 \mathrm{~A}$ ). Lysine-less $\mathrm{Ub}^{6}$ resembles unanchored, linear chains that have been proposed to regulate components of NF- $\mathrm{KB}$ in the mammalian system [53-55]; therefore, we posited that $\mathrm{Ub}^{6}-\mathrm{Stop}-\mathrm{K} 0 \mathrm{might}$ 
mimic endogenous poly-Ub involved in the Imd pathway and cause abnormal signaling, which could explain toxicity that we observe with the expression of $\mathrm{Ub}^{6}$-Stop-K0 chains (Figure 2).

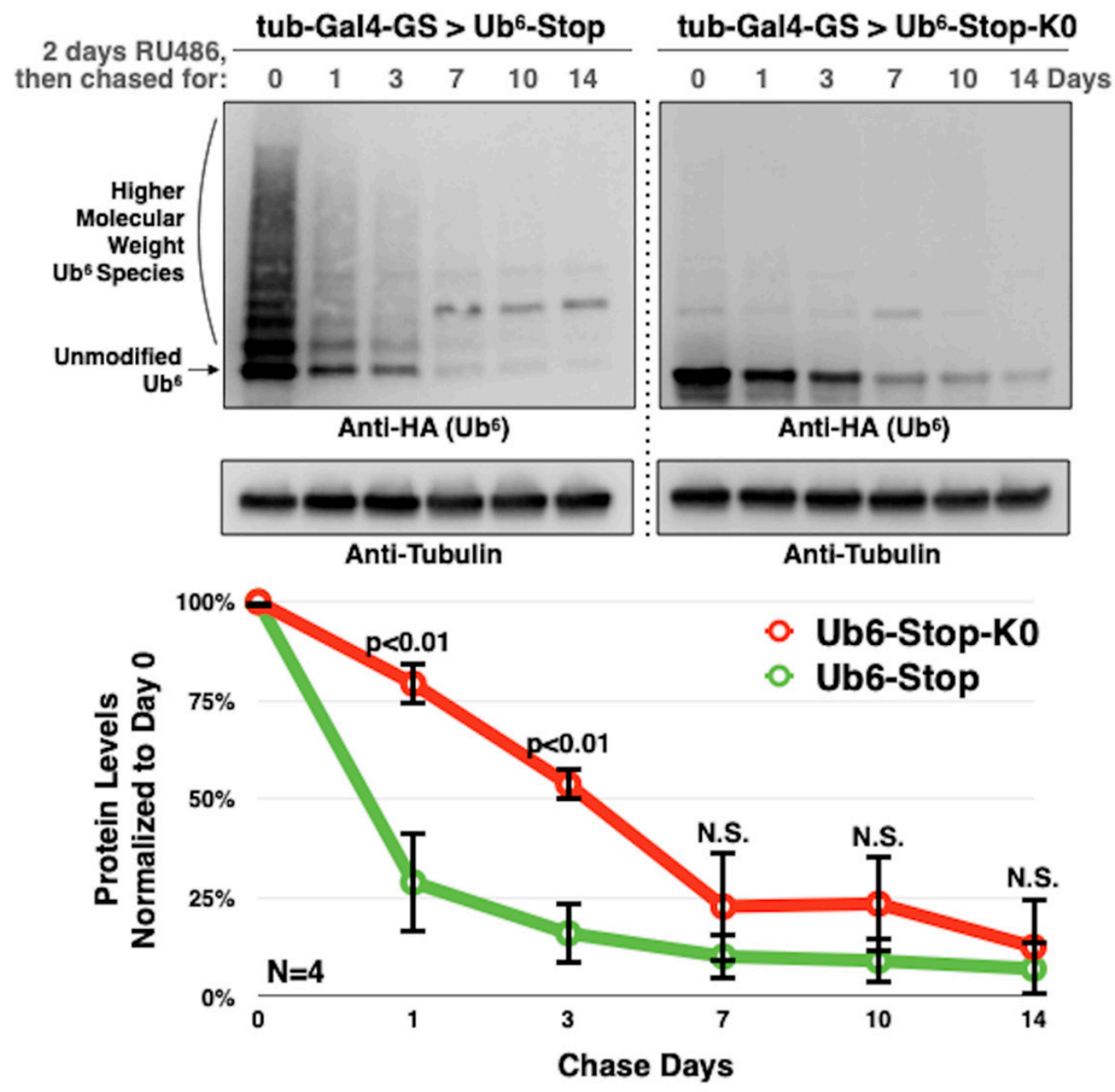

Figure 3. Decelerated turnover of lysine-less, non-cleavable ubiquitin chains in flies. Western blots from flies expressing the noted $\mathrm{Ub}^{6}$ transgenes under the RU486-dependent driver, tub-Gal4-GS for 2 days, and then "chased" in the absence of the inducer for up to 14 days. Whole-fly lysates. Graphs in the bottom are quantifications from images on the top and other independent repeats. The entire signal for each lane was quantified. Shown are means \pm standard deviation. ' $p$ ' values are from Student's $t$-tests comparing $\mathrm{Ub}^{6}$-Stop-K0 to $\mathrm{Ub}^{6}$-Stop of the same timepoint. "N.S." denotes no statistical significance.

To address the possibility of aberrant NF- $\mathrm{KB}$ signaling in the toxicity of lysine-less $\mathrm{Ub}^{6}$, we used the repo-Gal4 driver to express in glia $\mathrm{Ub}^{6}$-Stop- $\mathrm{K} 0$ alongside $\mathrm{RNAi}$ transgenes targeting components of the Drosophila Imd and Toll pathways, and monitored effects on longevity. We chose repo-Gal4 because $\mathrm{Ub}^{6}$-Stop-K0 expression in glial cells causes toxicity in developing and adult flies (Figure 2A,C); thus, any relief of this toxicity is observable along developmental and adult stages.

Independent knockdown of Relish and Dif significantly alleviates toxicity from $\mathrm{Ub}^{6}$-Stop-K0, although neither restores adult fly lifespan to that of the controls that do not express $\mathrm{Ub}^{6}$-Stop-K0 (Figure 4B). A separate longevity study revealed that knockdown of dorsal, cactus and IKK $\beta$ also significantly reduces toxicity (Figure $4 \mathrm{C}$ ). In this second study, $\mathrm{Ub}^{6}$-Stop-K0 leads to widespread pharate adult lethality with no surviving adults (Figure 2A summarizes fly stages of development). We have observed that transient factors, such as variation in ingredient lot numbers, can influence the development and survival of these flies; therefore, all comparisons in development and longevity are 
made only among flies reared simultaneously. Still, even though we notice some variation in the extent of toxicity, $\mathrm{Ub}^{6}$-Stop-K0 is consistently toxic and knockdown of NF-kB components consistently leads to increased survival (Figure 4B,C). The one exception is kenny. Knockdown of kenny does not improve toxicity from $\mathrm{Ub}^{6}$-Stop-K0. No flies with pan-glial expression of both kenny RNAi and $\mathrm{Ub}^{6}$-Stop-K0 survive to adulthood, despite the eclosure of a small number of flies expressing $\mathrm{Ub}^{6}$-Stop-K0 alone (Figure 4B). This observation indicates that the toxicity-rescuing effects we see with NF-kB knockdown are specific to key genes. In summary, targeting of several NF- $\mathrm{kB}$ players reduces the toxicity of lysine-less, free $\mathrm{Ub}^{6}$, implicating $\mathrm{Ub}^{6}$-Stop-K0 in immune signaling via both Imd and Toll pathways.
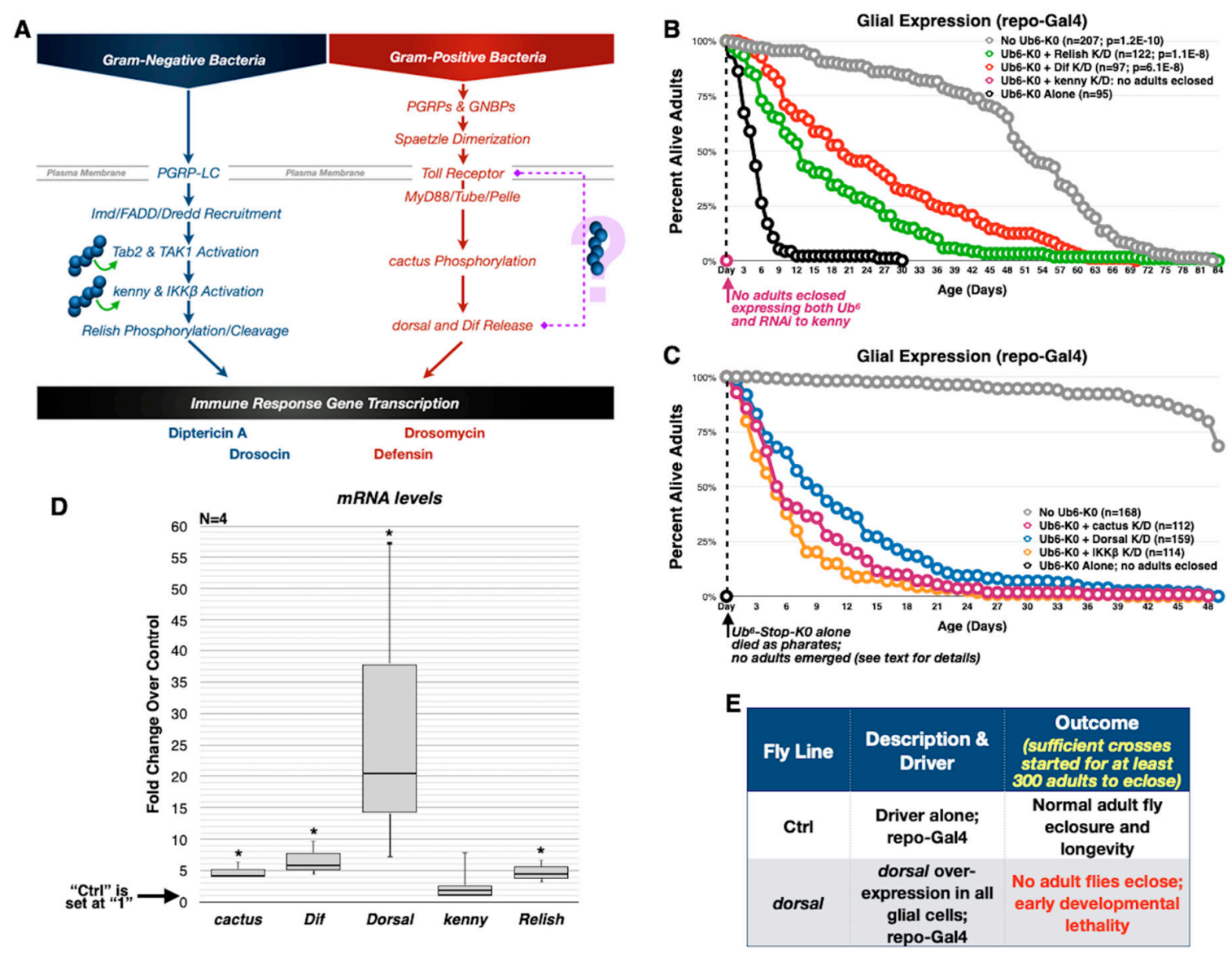

Figure 4. NF-kB pathway dysregulation when lysine-less, untethered hexa-ubiquitin is expressed in fly glia. (A) Summary of the IMD (left) and Toll (right) pathways in flies and potential roles for unanchored ubiquitin chains in them. (B,C) Longevity of adult flies expressing $\mathrm{Ub}^{6}$-Stop-K0 alone or in combination with RNAi-dependent knockdown of the noted NF-kB-related genes in fly glia. ' $p$ ' values are from log-rank tests. Longevity curves in panel B for " $\mathrm{No} \mathrm{Ub}^{6}-\mathrm{K} 0$ " and " $\mathrm{Ub}{ }^{6}-\mathrm{K} 0$ Alone" are the same as in Figure 2C. Adult flies from experiments with RNAi lines were collected and aged simultaneously and supplement results from earlier set ups where no adults emerged from repo-Gal4 $\mathrm{X} \mathrm{Ub}^{6}$-Stop-K0 crosses conducted for Figure 2C (all developing flies from those earlier crosses died as pharate adults). (D) Box-and-whisker plot of mRNA levels of the noted NF-kB-related genes by qRT-PCR from dissected heads of pharate adults expressing $\mathrm{Ub}^{6}$-Stop-K0 in glial cells, presented as fold change over controls. Statistics are from Student's $t$-tests comparing levels of each gene from Ub ${ }^{6}-\mathrm{Stop}-\mathrm{K} 0$ flies to "Ctrls" that contained the repo-Gal4 driver on the genetic background of $\mathrm{Ub}^{6}$-Stop-K0. Asterisks: $p<0.05$. (E) Survival outcomes for flies that express exogenous dorsal in glial cells, in the absence of any $\mathrm{Ub}^{6}$. 
To further explore this implication, we performed qRT-PCR using dissected heads from pharate adults expressing $\mathrm{Ub}^{6}$-Stop-K0 and repo-Gal4 to drive expression in glial cells only, compared to those with the same genetic background but without the $\mathrm{Ub}^{6}$ transgene. We used pharate adult heads because most $\mathrm{Ub}^{6}$-Stop-K0 adult flies do not eclose and because repo is robustly expressed in the head (flyatlas.com). In the presence of $\mathrm{Ub}^{6}$-Stop-K0, we observe statistically significantly increased levels of Relish, Cactus, Dif, and dorsal mRNA. In agreement with our previous results, kenny mRNA levels trend upward, however without reaching significance (Figure 4D). dorsal mRNA, in particular, is markedly upregulated. Importantly, overexpression of dorsal in glial cells recapitulates lethality in flies that do not express any $\mathrm{Ub}^{6}$, indicating that dorsal upregulation alone is sufficient for toxicity (Figure $4 \mathrm{E}$ ). These results illustrate a disturbance in normal NF- $\mathrm{KB}$ signaling in the presence of $\mathrm{Ub}^{6}$-Stop-K0 and present a mechanism for unanchored chain toxicity that relies on aberrant NF- $\mathrm{KB}$ upregulation.

\subsection{Aberrant NF-кB Signaling Is Mediated by the Ile44-Centered Hydrophobic Patch of Lysine-Less, Linear Poly-Ub}

Proper folding of $\mathrm{Ub}$ molecules results in a hydrophobic patch centered around amino acids isoleucine (ile) 44 , leucine 8 , histidine 68 , and valine 70 (Figure 5A; [5]). This surface is the recognition site for many $\mathrm{Ub}$-binding domains (UBDs) and is thus essential to the interaction of $\mathrm{Ub}$ with other proteins and for many of its actions (Figure 5B; [30-32,56]). NEMO, the NF- $\mathrm{kB}$ regulator, binds linear and lys63-linked chains [54,57] and 3D analysis of this interaction shows a critical position for Ub's ile44 (Figure 5C). Likewise, TAB2 and TAB3, which activate TAK1 in mammalian NF- $\kappa B$ signaling, recognize the ile44 patch through their C-terminal Np14 zinc finger domains [58].

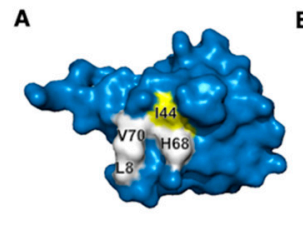

C
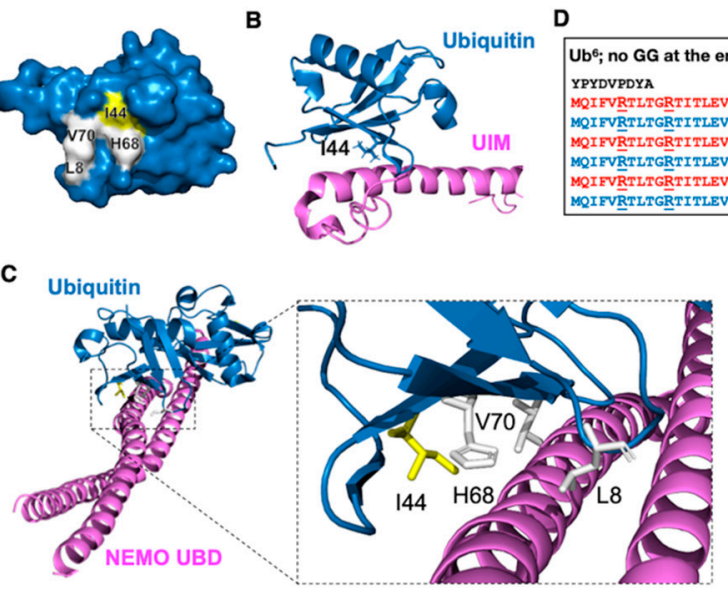

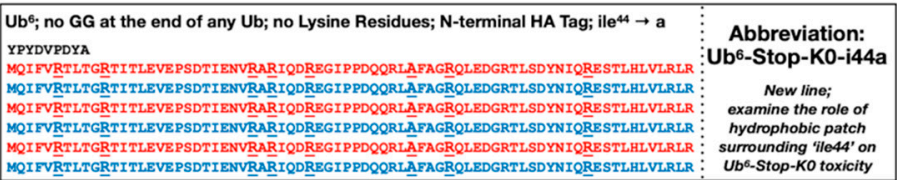

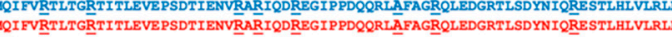
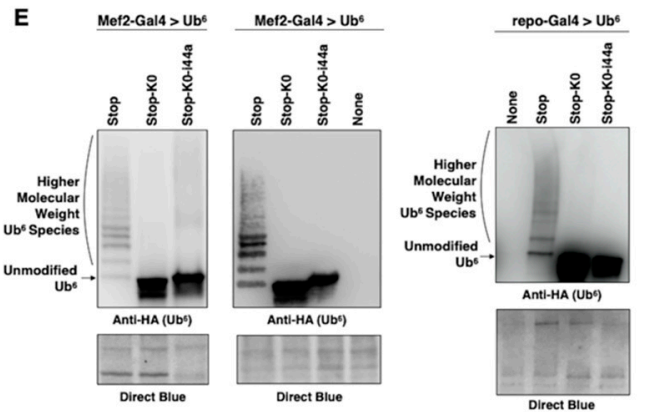

Figure 5. New transgenic, lysine-less ubiquitin chains with mutated isoleucine 44. (A) Surface representation of ubiquitin (PDB 1ubq) highlighting the structural location of the amino acids forming the hydrophobic patch involved in ubiquitin recognition (I44 in yellow, and L8, H68, and V70 in white). (B,C) Ribbon representation of ubiquitin (blue) displaying isoleucine 44 in the binding surface of different ubiquitin-interacting motifs in pink. (B, UIM; PDB 1yx5; C, the UBD domain of NEMO; PDB 2zvo). Inset highlights ubiquitin's hydrophobic patch and the key position of isoleucine 44 (yellow). Images were generated using PyMol (Schrodinger, Inc., New York, NY, USA). (D) Summary of the new ile44a transgenic line that we generated, its abbreviation and purpose. (E) Western blots from flies expressing the noted transgenes in all muscle cells (Mef2-Gal4, adult lysates) or all glial cells (repo-Gal4, pharate adult lysates).

Reasoning that ile44 within $\mathrm{Ub}^{6}$-Stop-K0 is likely important for its incorporation into, or recognition by, NF- $\mathrm{KB}$ signaling, we generated an additional $\mathrm{Ub}^{6}$ chain identical to $\mathrm{Ub}^{6}-\mathrm{Stop}-\mathrm{K} 0$, with the exception that every ile44 residue is mutated to alanine ( $\mathrm{Ub}^{6}-\mathrm{Stop}-\mathrm{K} 0$-ile44a; Figure $\left.5 \mathrm{D}\right)$, a mutation that is 
commonly used to disrupt the hydrophobic patch [30-32]. These new chains allow us to examine the influence of the ile44 hydrophobic patch on the toxicity of $\mathrm{Ub}^{6}$ chains. Expression of $\mathrm{Ub}^{6}$-Stop-K0-ile44a in Drosophila muscle cells and in glial cells leads to protein levels that are comparable to those of $\mathrm{Ub}^{6}$-Stop-K0. Similar to $\mathrm{Ub}^{6}$-Stop-K0, Ub ${ }^{6}$-Stop-K0-ile44a does not show the distinct HMW bands consistent with ubiquitination in Western blots. This is contrary to $\mathrm{Ub}^{6}$-Stop, which presents with clear and distinct laddering of ubiquitination above its main band (Figure 5E).

To assess toxicity from $\mathrm{Ub}^{6}$-Stop-K0-ile44a, we used the tissue-specific drivers described earlier to express the new transgene and then performed longevity assays. In each case, $\mathrm{Ub}^{6}$-Stop-K0-ile44a flies survive longer than their $\mathrm{Ub}^{6}$-Stop-K0 counterparts (Figure 6A,B). In fact, $\mathrm{Ub}^{6}$-Stop-K0-ile44a expression in muscle cells and in neurons does not have a statistically significant effect on adult fly lifespan compared to controls that express no $\mathrm{Ub}^{6}$. Driving expression in all fly cells or only in glia results in a modest reduction in longevity (Figure 6B). It is particularly remarkable that disrupting the hydrophobic interaction interface of $\mathrm{Ub}$ eliminates developmental lethality from ubiquitous expression of $\mathrm{Ub}^{6}$-Stop- $\mathrm{K} 0$ and only mildly reduces adult fly longevity compared to controls without $\mathrm{Ub}^{6}$ (Figure 6A,B).

Next, we examined whether reduced toxicity from $\mathrm{Ub}^{6}$-Stop-K0-ile44a coincides with normalized levels of genes involved in NF- $\kappa B$ signaling, compared to $\mathrm{Ub}^{6}$-Stop-K0. We recapitulated studies conducted for Figure $4 \mathrm{D}$ by using new crosses and adding flies that express $\mathrm{Ub}^{6}$-Stop-K0-ile44a in all glia. As summarized in qRT-PCR results in Figure 6C, the mRNA levels of dorsal and Relish are not statistically different in the presence of $\mathrm{Ub}^{6}$-Stop-K0-ile44a compared to flies not expressing any $\mathrm{Ub}^{6}$ transgenes, but continue to be upregulated in the presence of $\mathrm{Ub}^{6}$-Stop-K0.

We validated these findings by examining the protein levels of Relish (antibodies tested for other NF- $\mathrm{kB}$ components in flies were non-specific or inconsistent). We observed that in the presence of $\mathrm{Ub}^{6}$-Stop-K0, Relish protein levels are noticeably higher, compared to flies not expressing any $\mathrm{Ub}^{6}$ transgene, or expressing the ubiquitinatable $\mathrm{Ub}^{6}$-Stop. In the presence of $\mathrm{Ub}^{6}$-Stop-K0-ile44a, Relish levels return towards normality (Figure $6 \mathrm{D}$ ). Because NF- $\mathrm{kB}$ transcription factors translocate to the nucleus when activated, we performed subcellular fractionation to visualize nuclear Relish protein levels in heads dissected from pharate adults expressing $\mathrm{Ub}^{6}$ in glial cells. We observed that more Relish is present in the nuclei of $\mathrm{Ub}^{6}$-Stop-K0 pharate adults, compared to controls that are genetically similar, but lack $\mathrm{Ub}^{6}$ (Figure 6E). Nuclear Relish levels in the presence of either $\mathrm{Ub}^{6}$-Stop or $\mathrm{Ub}^{6}$-Stop-K0-ile44a appear lower than those in the presence of $\mathrm{Ub}^{6}$-Stop-K0, indicating the importance of the chains' susceptibility to ubiquitination and ile44. Collectively, these results lead us to conclude that ubiquitination-resistant, free, linear $\mathrm{Ub}$ chains can be highly toxic in flies at least in part due to NF- $\mathrm{KB}$ signaling that is dependent on the ile44-centered hydrophobic patch on $\mathrm{Ub}$.

Due to a lack of quality antibodies for NF- $\mathrm{KB}$ components in flies, we performed subcellular fractionation experiments using cultured, HEK-293T human cells to validate our findings in Figure 6E. In humans, p65 is an NF- $\mathrm{kB}$ transcription factor whose phosphorylation at serine 536 controls its nuclear translocation and activity [59]. To examine p65 translocation, we transiently transfectedHEK-293T cells with the $\mathrm{Ub}^{6}$ constructs and, $24 \mathrm{~h}$ later, performed subcellular fractionation and Western blotting. Expression of the various $\mathrm{Ub}^{6}$ constructs in HEK-293T cells leads to ubiquitinated species for $\mathrm{Ub}^{6}$-Stop, but not so for lysine-less variants (Figure 7A). As shown in Figure 7B, $\mathrm{Ub}^{6}$-Stop-K0 expression leads to markedly more endogenous phosphorylated-p65 in the nucleus compared to cells transfected with empty vector; the ile44a mutation reverses this effect. There was no statistically significant difference in the total p65 levels in the cytoplasm or nucleus (Figure 7B). Based on the increased nuclear translocation of activated $\mathrm{p} 65$, it is likely that $\mathrm{Ub}^{6}$-Stop-K0 induces the transcription of downstream NF- $\mathrm{kB}$ response genes; additional studies are needed to determine the precise outcomes. These results reinforce a role for ubiquitination-resistant, unanchored, linear poly-Ub in NF-kB signaling, notably in a human cell line. 
A

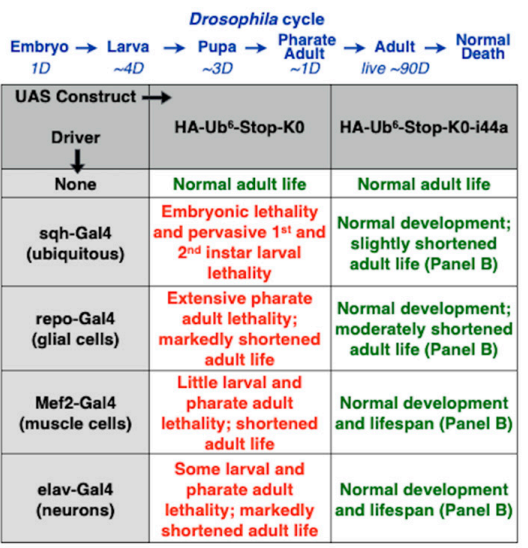

C

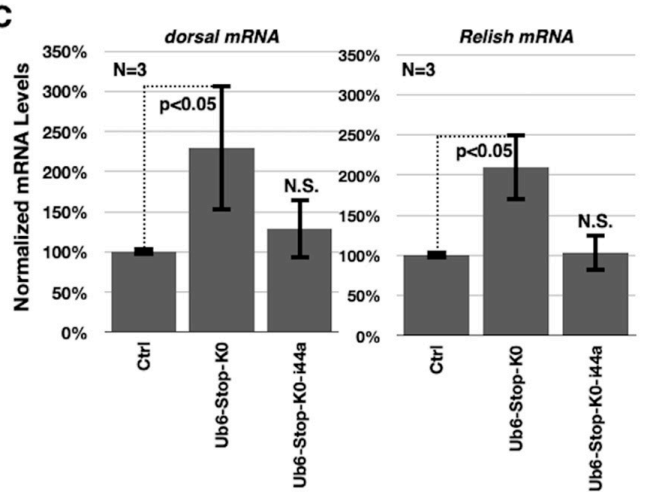

B

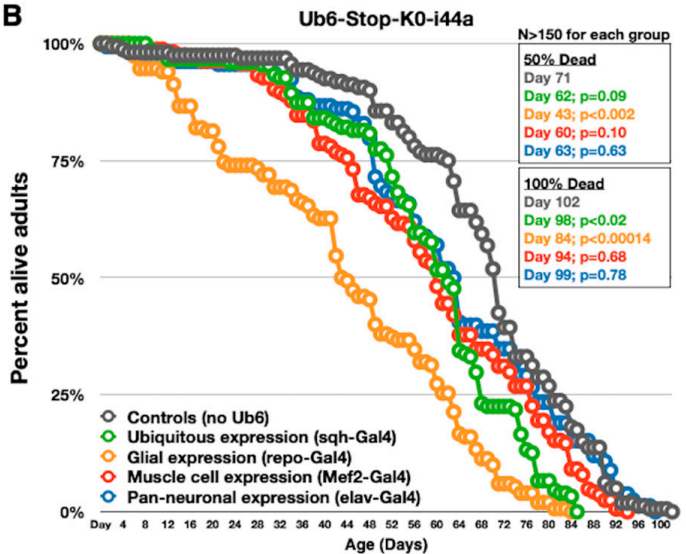

D

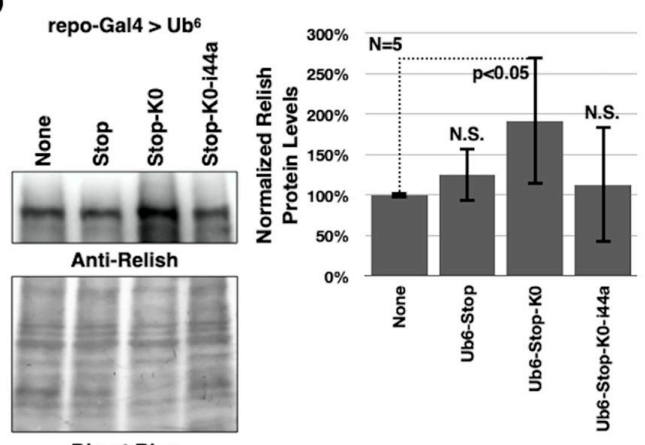

E

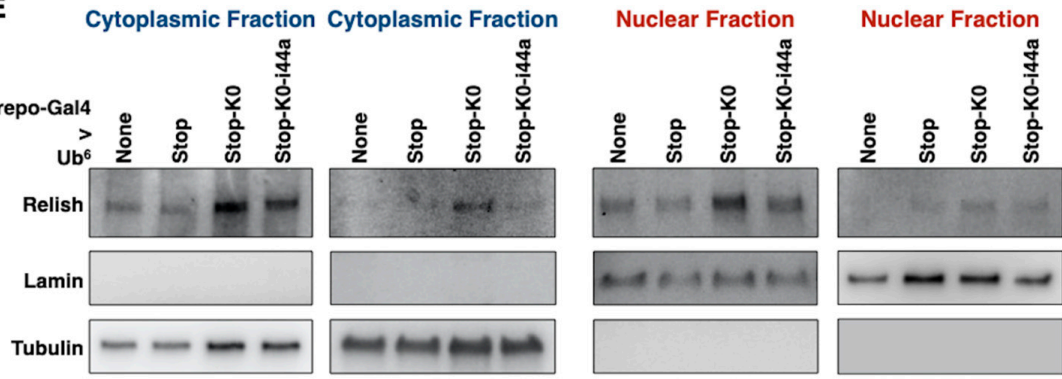

Figure 6. Isoleucine 44 mediates toxicity from free, linear, lysine-less ubiquitin chains. (A) Summary of findings when the noted transgenes are expressed as described. "None" denotes no Gal4 driver was present. Results are from crosses that were conducted simultaneously. (B) Longevity assays of adult flies expressing $\mathrm{Ub}^{6}$ chains under the noted Gal4 drivers. "Ctrls" do not contain $\mathrm{Ub}^{6}$ transgenes, but have the Gal4 driver on the genetic background that we utilized to generate the $\mathrm{Ub}^{6}$ chain-containing flies. ' $p$ ' values are from log-rank tests. (C) mRNA levels of the noted NF-kB-related genes by qRT-PCR in dissected heads of pharate adults expressing $\mathrm{Ub}^{6}-\mathrm{Stop}-\mathrm{K} 0$ or $^{\mathrm{Ub}} \mathrm{b}^{6}$-Stop-K0-i44a in glial cells, presented as fold change over controls without the $\mathrm{Ub}^{6}$ transgene. ' $p$ ' values are from Student's $t$-tests comparing $\mathrm{Ub}^{6}$-expressing flies to controls. (D) Western blots from dissected pharate adult heads expressing the noted transgenes in all glial cells (left). Relish protein levels were normalized to direct blue and quantified (right). ' $p$ ' value is from Student's $t$-tests comparing $\mathrm{Ub}^{6}$-expressing flies to controls that contain the driver on the same genetic background as ubiquitin chain-encoding flies. (E) Western blots from two independent cytoplasmic/nuclear fractionations of dissected pharate adult heads expressing, or not, $\mathrm{Ub}^{6}$ transgenes in glial cells. In (C,D), "N.S." denotes no statistical significance comparing a specific $\mathrm{Ub}^{6}$ group to its respective control. Due to the temporary closure of our institution as a result of the COVID-19 pandemic, we were unable to obtain additional, independent samples for panel (E). We also encountered some difficulties with signal-to-noise ratio from the anti-Relish antibody for panel (E), but were unable to procure more reagents as a result of university closure. 


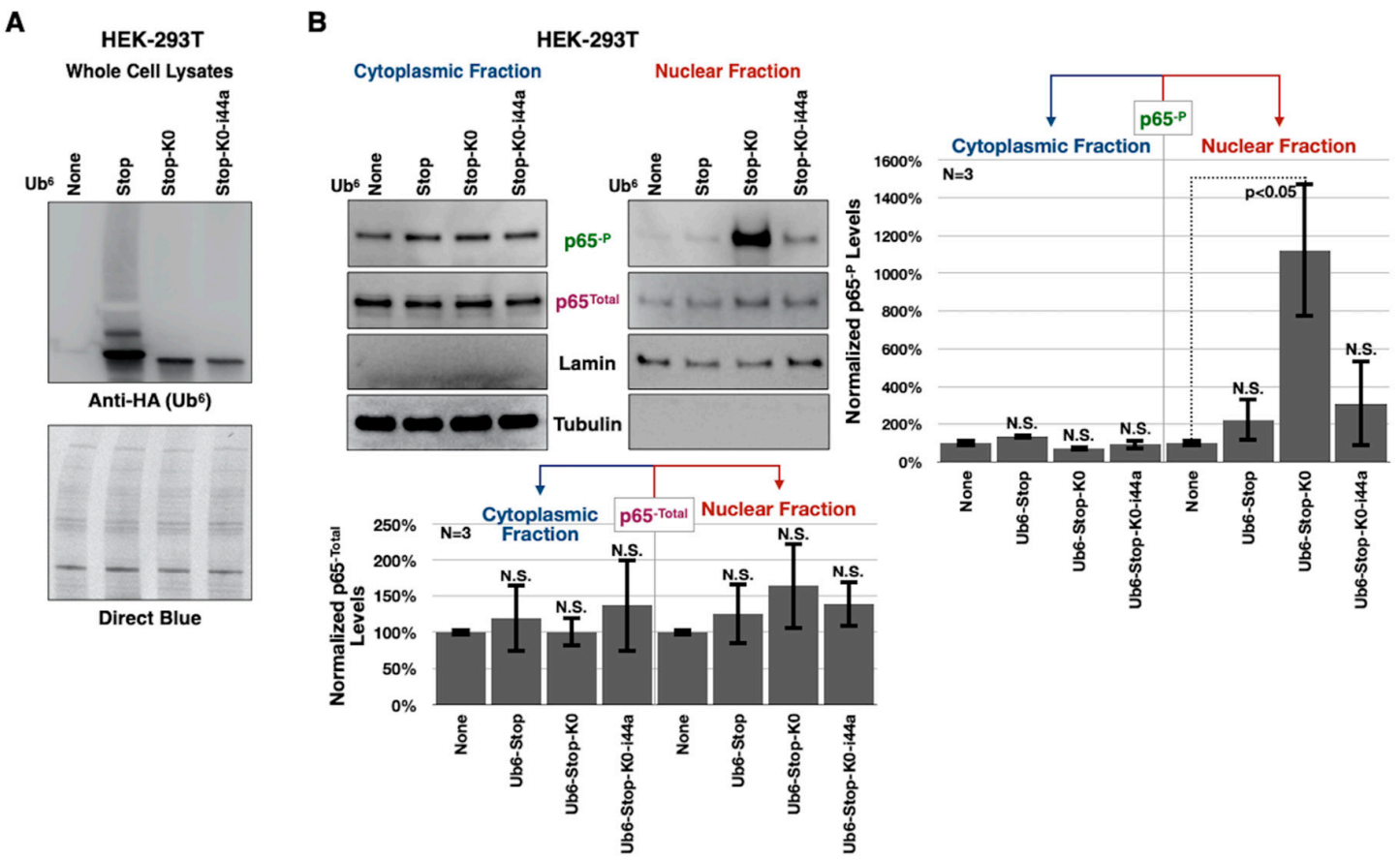

Figure 7. Isoleucine 44 mediates nuclear translocation of phosphorylated p65 in mammalian cells. (A) Western blots from whole-cell lysates of HEK-293T cells transfected with empty vector or $\mathrm{Ub}^{6}$ plasmids. (B) Western blots and quantifications from cytoplasmic/nuclear fractionation of HEK-293T cells transfected as in (A). For both $(\mathbf{A}, \mathbf{B})$, results are representative of three independent experiments. In panel (B), p65 protein levels were quantified, divided by the respective loading control (tubulin or lamin) for each fraction and normalized to controls lacking $\mathrm{Ub}^{6}$. Shown in histograms are means \pm standard deviation. ' $p$ ' value is from Student's $t$-tests comparing cells expressing $\mathrm{Ub}^{6}$ to non-Ub $\mathrm{Ub}^{6}$-expressing controls. "N.S." denotes no statistical significance comparing a specific $\mathrm{Ub}^{6}$ group to its respective control. p65-P : phosphorylated-p65. The COVID-19 situation also hampered our ability to conduct additional cell-based studies.

\section{Discussion}

We have developed a model to study the control and function of untethered poly- $\mathrm{Ub}$ in an intact, multicellular organism, Drosophila melanogaster. By manipulating the properties of linear, unanchored poly- $\mathrm{Ub}$, we propose that free chains are not intrinsically toxic, but that the ability of cells to control them, e.g., through endogenous ubiquitination, is crucial to their remaining innocuous. $\mathrm{Ub}^{6}$-Stop-K0, which is unreceptive to ubiquitination, causes aberrant NF- $\mathrm{kB}$ signaling and fly death that is largely dependent on amino acid ile44 on $\mathrm{Ub}$. Our results, therefore, also demonstrate a critical role for ile44 on $\mathrm{Ub}$ in NF-kB-dependent pathways.

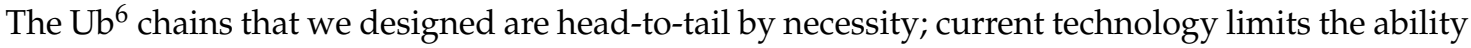
to stably, genetically encode other linkage types in vivo. Endogenous, unanchored poly-Ub chains are diverse in their linkage types and lengths, and their composition controls their functions and interaction partners $[5,10,12,16,51]$. We previously characterized $\mathrm{Ub}^{6}$ species that are endogenously ubiquitinated, transforming them into branched chains with various lysine linkages [26]. Unlike them, $\mathrm{Ub}^{6}$-Stop-K0 and $\mathrm{Ub}^{6}$-Stop-K0-ile44a that we described here are homogenous, linear poly-Ub. We also established in prior work [26] and through the results of this study that each of the $\mathrm{Ub}^{6}$ species has distinct properties, from turnover rates to physiological outcomes. For these reasons, the transgenically-encoded $\mathrm{Ub}^{6}$ fly lines that we developed serve as valuable tools to study the function and regulation of unanchored poly-Ub in vivo.

It remains to be resolved which proteins recognize and interact with $\mathrm{Ub}^{6}$-Stop-K0. For example, upstream elements that lead to upregulated NF- $\mathrm{kB}$ component levels in the presence of lysine-less, 
linear poly-Ub need to be identified. Still, we infer that these elements likely depend on interacting with the ile 44 hydrophobic patch, since mutating this amino acid residue markedly reverses $\mathrm{Ub}^{6}$-Stop-K0 toxicity in intact flies. Our results highlight NF- $\mathrm{kB}$ components as key players in toxicity from ubiquitination-resistant, linear chains. According to mammalian studies, canonical activation of NF- $\mathrm{kB}$ requires the linear $\mathrm{Ub}$ chain assembly complex (LUBAC). LUBAC attaches head-to-tail, met1-linked chains to NEMO, an NF- $\mathrm{KB}$ regulator, and participates in the synthesis of branched, unanchored poly-Ub chains composed of both met1- and lys63-linkages [16]. Free Ub chains are required for the activation of the MAP kinase kinase kinase, TAK1 and the subsequent phosphorylation and degradation of the inhibitory kinase, IKK $\beta$ [50]. Several other proteins within the pathway, including NEMO and the LUBAC proteins, contain UBDs that may interact with untethered chains and may be regulated by them [49]. Thus, it is likely that the presence of lysine-less, linear, unanchored poly-Ub places NF- $\mathrm{KB}$ in overdrive by interacting with NEMO and LUBAC. In the absence of a functional binding interface on $\mathrm{Ub}$ (the ile44a mutation), NF- $\mathrm{kB}$ is not improperly triggered. Our results lead to the conclusion that toxicity induced by linear chains is due to overstimulation of the NF- $\mathrm{KB}$ pathway. In the case of the ile44a mutant, these linear chains are not recognizable by the cellular components so their expression per se does not induce toxicity.

As introduced earlier, flies have two NF-kB pathways, Imd and Toll. The Imd pathway is initiated when Gram-negative bacteria are detected by peptidoglycan recognition protein-LC at the plasma membrane [60-62]. This event triggers the activation of Tab2/Tak1 that phosphorylates and activates the IKK complex (kenny and IKK $\beta$ ) [63-65] which then, via Relish (the NF- $\mathrm{KB}$ transcription factor), activates antimicrobial genes including Diptericin and Cecropin ([66-68] and Figure 4A). The involvement of unanchored poly- $\mathrm{Ub}$ in the Imd pathway is expected at multiple steps, based on mammalian NF- $\mathrm{KB}$ signaling orthologues. Ubcd4, an E2 conjugase whose mammalian orthologue, E2-25K/Ube2K, generates poly-Ub chains without a substrate protein [69], is required for Imd activation [70]. The concomitant knockdown of the E3 ligase Bendless and the E2 conjugase Uev1a abrogates Imd signaling in cultured insect cells [71], and Bendless/Uev1a together synthesize unanchored lys63-linked $\mathrm{Ub}$ chains in vitro [72]. To the best of our knowledge, there are no published reports showing a direct involvement of unanchored poly-Ub in the Imd pathway in Drosophila, but the importance of Ubcd4 and Bendless/Uev1a implicates these chains. Our results that linear poly-Ub activates Relish strengthen this connection and further indicate that free, linear $\mathrm{Ub}$ chains are involved in the Imd pathway in flies.

Unlike for the Imd pathway, where evidence from mammalian orthologues and functional assays of fly proteins indicates a role for free $\mathrm{Ub}$ chains, it is unclear whether unanchored poly- $\mathrm{Ub}$ is involved in the Toll pathway. Toll signaling begins when Gram-positive bacteria or fungi are detected by extracellular peptidoglycan recognition proteins and glucan-binding proteins, triggering a cascade that results in the processing of the Toll ligand Spaetzle to facilitate its binding to the Toll receptor [73-75]. After activation, the Toll receptor binds the adaptor MyD88 and recruits Tube and Pelle to phosphorylate cactus [76], ultimately activating dorsal and Dif [77] and driving the transcription of antimicrobial peptides like Drosomycin and Defensin ([78] and Figure 4A). Insofar as we know, our data that free, linear poly-Ub increases levels of cactus and dorsal are the first to link linear chains to the Toll pathway, thus merging Gram-negative and Gram-positive/fungi, NF-kB-centered processes under the regulation of linear Ub chains. Which proteins serve as "receptors" that recognize lysine-less, linear poly-Ub and lead to activation of the Toll pathway? Studies with unbiased approaches and targeted genetics will be necessary to uncover these details and also to identify downstream genes that are impacted by free, lysine-less Ub chains for both the Imd and Toll pathways. Based on increased nuclear levels of Relish and phosphorylated-p65 proteins in flies and mammalian cells expressing $\mathrm{Ub}^{6}$-Stop- $\mathrm{K} 0$, there is good reason to predict a genetic response to these transcription factors.

The extent of NF- $\mathrm{KB}$ component perturbation by linear $\mathrm{Ub}$ chains differed among experiments in this work. For example, in Figure 6C, glial expression of $\mathrm{Ub}^{6}$-Stop-K0 did not induce dorsal mRNA expression to the magnitude shown in Figure 4D. As mentioned above, we have observed physiological effects from different fly media conditions, even using the same food recipe. Flies used in Figure 4 were 
reared on a lot of food that was different from those in Figure 6, which might have been more prone to mold or bacterial growth and could have itself induced background NF-KB signaling. We stress the importance of making comparisons only among flies and their respective controls that were maintained on the same batch of food and monitored at the same time. In our case, all flies from Figure 4D were reared together and collected independently of those in Figure 6C, which were reared and collected as a separate cohort. However, regardless of the observed experimental variation, the important point remains that the induction of both dorsal and Relish mRNA was consistent among flies reared simultaneously: $\mathrm{Ub}^{6}$-Stop-K0 significantly increases their levels, whereas $\mathrm{Ub}^{6}$-Stop-K0-ile44a does not have the same impact.

Our work provides new insight into Ub biology more generally. The current model of $\mathrm{Ub}$ utilization and recycling centers on the use of mono- $\mathrm{Ub}$ in a stepwise manner to generate poly- $\mathrm{Ub}$ and the disassembly of poly-Ub into mono-Ub before reuse (Figure 8). We previously described [26] and further evidence here the possibility of alternative routes for $\mathrm{Ub}$ use and recycling, based on the notion that free $\mathrm{Ub}$ chains may not need to be disassembled into mono-Ub to be eliminated. They can be conjugated en bloc onto other proteins, or can be degraded without the need of disassembly into their building blocks [26]. These routes can effectively recycle untethered poly-Ub, which seems largely innocuous to an intact organism, except under highly specific conditions, such as the ones in the current study (Figure 8). We propose that cells might employ various types of Ub utilization, recycling and disposal routes, depending on homeostatic needs. For example, conjugation of free poly-ub onto specific proteins without prior disassembly might be beneficial under times of energy stress or proteotoxic pressure, where quick and efficient removal of certain proteins might benefit from Ub chain "hopping". These and other possibilities deserve attention to understand Ub biology.

\section{Traditional Ub cycle}

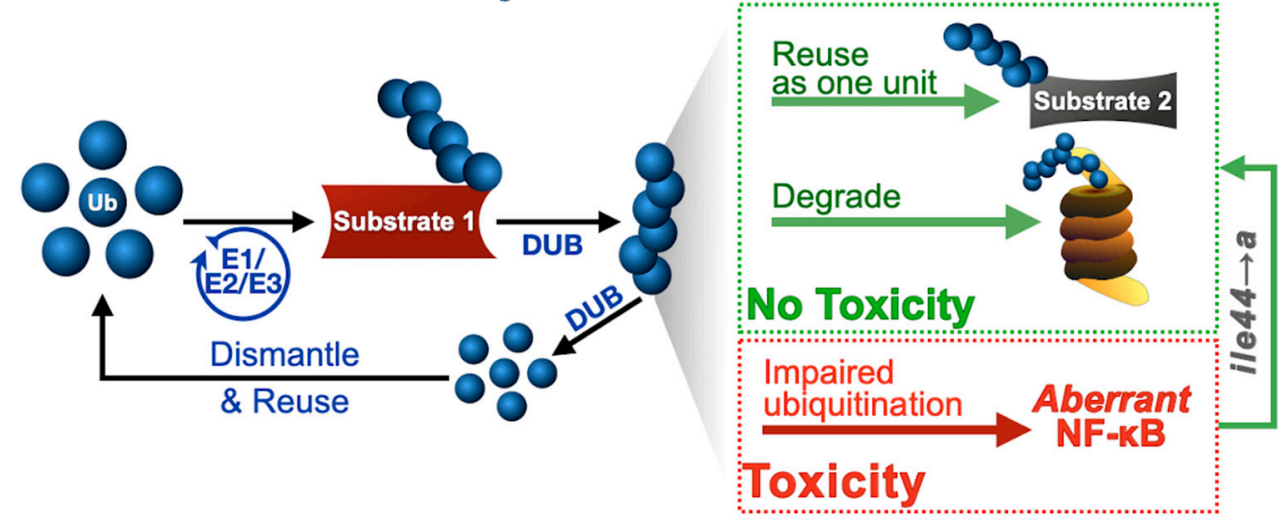

Figure 8. Summary of potential outcomes of ubiquitin recycling. "Traditional Ub cycle": ubiquitin is attached to a substrate protein either as a monomer (not shown) or as a polymer. Poly-ubiquitin can be removed as a single unit by DUBs, dismantled into mono-ubiquitin, and then reused. "Additional outcomes": based on our studies in Drosophila from earlier investigations and from this work, unanchored poly-ubiquitin can be reused as a single unit and become attached onto another protein [26], or can be degraded ([26] and this work). If handled as described thus far, unanchored chains are not detrimental (No Toxicity). However, if the ability of cells to reuse or to ubiquitinate free poly-ubiquitin as described here is hampered, unanchored chain toxicity results from cellular pathway perturbation, as with NF-kB processes evidenced here (Toxicity). Based on our data, this toxicity is reversed by an isoleucine 44 to alanine mutation that disrupts a critical interaction site on ubiquitin.

Drosophila is an excellent model organism due its flexible genetics, easy and inexpensive maintenance, low number of chromosomes, and short life cycle. Importantly, most genes and pathways are conserved among Drosophila and vertebrates, allowing for reliable translation of findings from flies to higher-order organisms [79]. It will be important to expand our findings in a mammalian system to 
examine the role of endogenous, unanchored poly- $\mathrm{Ub}$ in various disease models and stress responses. Free $\mathrm{Ub}$ chains are upregulated under certain stress conditions, including inflammation [18] —what types of unanchored chains might we see in mammals with inflammatory diseases? Are there tissue-specific differences? Are there disease-specific variations? Diversity in unanchored $\mathrm{Ub}$ chain signaling - from chain length to composition to available binding interfaces-will be important to consider when studying the effects of these species going forward.

In summary, our results reinforce the notion that free $\mathrm{Ub}$ chains are not inherently detrimental. Our investigations contribute to the overall understanding of $\mathrm{Ub}$ biology, recognize the ile44-centered hydrophobic patch of $\mathrm{Ub}$ as an important site for toxicity, and identify ways to reduce and prevent deleterious effects from untethered $\mathrm{Ub}$ chains in vivo (Figure 8). Continued work is necessary to probe into these possibilities in different organisms and under various physiological conditions.

Author Contributions: Conceptualization and methodology, J.R.B., G.M.S., and S.V.T.; formal analysis, J.R.B. G.M.S., and S.V.T.; investigation, J.R.B., K.L., and S.V.T.; data analysis and manuscript preparation, J.R.B., G.M.S., and S.V.T.; visualization, J.R.B., G.M.S., and S.V.T.; funding acquisition, J.R.B., G.M.S., and S.V.T. All authors have read and agreed to the published version of the manuscript.

Funding: This work was funded by a Thomas Rumble Graduate Fellowship to J.R.B. from Wayne State University Graduate School, by a Competitive Graduate Research Assistantship to J.R.B. from Wayne State University Graduate School, by R00ES025835 to G.M.S. from the National Institute of Environmental Health Sciences, and by R01NS086778 to S.V.T. from the National Institute of Neurological Disorders and Stroke.

Acknowledgments: We thank K. Matthew Scaglione (Duke University) for critical and insightful discussions of this work, Jamie Roebuck (Duke University) for helping with transgenic fly generation and the Developmental Studies Hybridoma Bank for antibodies.

Conflicts of Interest: The authors declare no conflict of interest.

\section{References}

1. Stallcup, M.R. Role of protein methylation in chromatin remodeling and transcriptional regulation. Oncogene 2001, 20, 3014-3020. [CrossRef]

2. Canagarajah, B.J.; Khokhlatchev, A.; Cobb, M.H.; Goldsmith, E.J. Activation mechanism of the MAP kinase ERK2 by dual phosphorylation. Cell 1997, 90, 859-869. [CrossRef]

3. Lis, H.; Sharon, N. Protein glycosylation. Structural and functional aspects. Eur. J. Biochem. 1993, $218,1-27$. [CrossRef]

4. Thrower, J.S.; Hoffman, L.; Rechsteiner, M.; Pickart, C.M. Recognition of the polyubiquitin proteolytic signal. EMBO J. 2000, 19, 94-102. [CrossRef] [PubMed]

5. Komander, D.; Rape, M. The ubiquitin code. Annu. Rev. Biochem. 2012, 81, 203-229. [CrossRef] [PubMed]

6. Todi, S.V.; Scaglione, K.M.; Blount, J.R.; Basrur, V.; Conlon, K.P.; Pastore, A.; Elenitoba-Johnson, K.; Paulson, H.L. Activity and cellular functions of the deubiquitinating enzyme and polyglutamine disease protein ataxin-3 are regulated by ubiquitination at lysine 117. J. Biol. Chem. 2010, 285, 39303-39313. [CrossRef]

7. Todi, S.V.; Winborn, B.J.; Scaglione, K.M.; Blount, J.R.; Travis, S.M.; Paulson, H.L. Ubiquitination directly enhances activity of the deubiquitinating enzyme ataxin-3. EMBO J. 2009, 28, 372-382. [CrossRef]

8. Tsou, W.L.; Burr, A.A.; Ouyang, M.; Blount, J.R.; Scaglione, K.M.; Todi, S.V. Ubiquitination regulates the neuroprotective function of the deubiquitinase ataxin-3 in vivo. J. Biol. Chem. 2013, 288, 34460-34469. [CrossRef]

9. Ristic, G.; Tsou, W.L.; Todi, S.V. An optimal ubiquitin-proteasome pathway in the nervous system: The role of deubiquitinating enzymes. Front. Mol. Neurosci. 2014, 7, 72. [CrossRef]

10. Yau, R.; Rape, M. The increasing complexity of the ubiquitin code. Nat. Cell Biol. 2016, 18, 579-586. [CrossRef] [PubMed]

11. Yau, R.G.; Doerner, K.; Castellanos, E.R.; Haakonsen, D.L.; Werner, A.; Wang, N.; Yang, X.W.; Martinez-Martin, N.; Matsumoto, M.L.; Dixit, V.M.; et al. Assembly and Function of Heterotypic Ubiquitin Chains in Cell-Cycle and Protein Quality Control. Cell 2017, 171, 918-933.e92. [CrossRef] [PubMed]

12. Swatek, K.N.; Komander, D. Ubiquitin modifications. Cell Res. 2016, 26, 399-422. [CrossRef] [PubMed]

13. Clague, M.J.; Coulson, J.M.; Urbe, S. Cellular functions of the DUBs. J. Cell Sci. 2012, 125, 277-286. [CrossRef] [PubMed] 
14. Komander, D.; Clague, M.J.; Urbe, S. Breaking the chains: Structure and function of the deubiquitinases. Nat. Rev. Mol. Cell Biol. 2009, 10, 550-563. [CrossRef] [PubMed]

15. Reyes-Turcu, F.E.; Shanks, J.R.; Komander, D.; Wilkinson, K.D. Recognition of polyubiquitin isoforms by the multiple ubiquitin binding modules of isopeptidase T. J. Biol. Chem. 2008, 283, 19581-19592. [CrossRef] [PubMed]

16. Emmerich, C.H.; Ordureau, A.; Strickson, S.; Arthur, J.S.; Pedrioli, P.G.; Komander, D.; Cohen, P. Activation of the canonical IKK complex by K63/M1-linked hybrid ubiquitin chains. Proc. Natl. Acad. Sci. USA 2013, 110, 15247-15252. [CrossRef]

17. Hao, R.; Nanduri, P.; Rao, Y.; Panichelli, R.S.; Ito, A.; Yoshida, M.; Yao, T.P. Proteasomes activate aggresome disassembly and clearance by producing unanchored ubiquitin chains. Mol. Cell. 2013, 51, 819-828. [CrossRef]

18. Braten, O.; Shabek, N.; Kravtsova-Ivantsiv, Y.; Ciechanover, A. Generation of free ubiquitin chains is up-regulated in stress and facilitated by the HECT domain ubiquitin ligases UFD4 and HUL5. Biochem. J. 2012, 444, 611-617. [CrossRef]

19. Grou, C.P.; Pinto, M.P.; Mendes, A.V.; Domingues, P.; Azevedo, J.E. The de novo synthesis of ubiquitin: Identification of deubiquitinases acting on ubiquitin precursors. Sci. Rep. 2015, 5, 12836. [CrossRef]

20. Kovacs, L.; Nagy, O.; Pal, M.; Udvardy, A.; Popescu, O.; Deak, P. Role of the deubiquitylating enzyme DmUsp5 in coupling ubiquitin equilibrium to development and apoptosis in Drosophila melanogaster. PLoS ONE 2015, 10, e0120875. [CrossRef]

21. Clague, M.J.; Barsukov, I.; Coulson, J.M.; Liu, H.; Rigden, D.J.; Urbe, S. Deubiquitylases from genes to organism. Physiol. Rev. 2013, 93, 1289-1315. [CrossRef] [PubMed]

22. Wang, C.H.; Chen, G.C.; Chien, C.T. The deubiquitinase Leon/USP5 regulates ubiquitin homeostasis during Drosophila development. Biochem. Biophys. Res. Commun. 2014, 452, 369-375. [CrossRef] [PubMed]

23. Amerik, A.; Swaminathan, S.; Krantz, B.A.; Wilkinson, K.D.; Hochstrasser, M. In vivo disassembly of free polyubiquitin chains by yeast Ubp14 modulates rates of protein degradation by the proteasome. EMBO J. 1997, 16, 4826-4838. [CrossRef] [PubMed]

24. Piotrowski, J.; Beal, R.; Hoffman, L.; Wilkinson, K.D.; Cohen, R.E.; Pickart, C.M. Inhibition of the $26 \mathrm{~S}$ proteasome by polyubiquitin chains synthesized to have defined lengths. J. Biol. Chem. 1997, 272, 23712-23721. [CrossRef]

25. Dayal, S.; Sparks, A.; Jacob, J.; Allende-Vega, N.; Lane, D.P.; Saville, M.K. Suppression of the deubiquitinating enzyme USP5 causes the accumulation of unanchored polyubiquitin and the activation of p53. J. Biol. Chem. 2009, 284, 5030-5041. [CrossRef]

26. Blount, J.R.; Libohova, K.; Marsh, G.B.; Sutton, J.R.; Todi, S.V. Expression and Regulation of Deubiquitinase-Resistant, Unanchored Ubiquitin Chains in Drosophila. Sci. Rep. 2018, 8, 8513. [CrossRef]

27. Blount, J.R.; Meyer, D.N.; Akemann, C.; Johnson, S.L.; Gurdziel, K.; Baker, T.R.; Todi, S.V. Unanchored ubiquitin chains do not lead to marked alterations in gene expression in Drosophila melanogaster. Biol. Open 2019, 8, bio043372. [CrossRef]

28. Amerik, A.Y.; Hochstrasser, M. Mechanism and function of deubiquitinating enzymes. Biochim. Biophys. Acta 2004, 1695, 189-207. [CrossRef]

29. Dikic, I.; Wakatsuki, S.; Walters, K.J. Ubiquitin-binding domains - from structures to functions. Nat. Rev. Mol. Cell Biol. 2009, 10, 659-671. [CrossRef]

30. Beal, R.; Deveraux, Q.; Xia, G.; Rechsteiner, M.; Pickart, C. Surface hydrophobic residues of multiubiquitin chains essential for proteolytic targeting. Proc. Natl. Acad. Sci. USA 1996, 93, 861-866. [CrossRef]

31. Beal, R.E.; Toscano-Cantaffa, D.; Young, P.; Rechsteiner, M.; Pickart, C.M. The hydrophobic effect contributes to polyubiquitin chain recognition. Biochemistry 1998, 37, 2925-2934. [CrossRef] [PubMed]

32. Sloper-Mould, K.E.; Jemc, J.C.; Pickart, C.M.; Hicke, L. Distinct functional surface regions on ubiquitin. J. Biol. Chem. 2001, 276, 30483-30489. [CrossRef] [PubMed]

33. Fish, M.P.; Groth, A.C.; Calos, M.P.; Nusse, R. Creating transgenic Drosophila by microinjecting the site-specific phiC31 integrase mRNA and a transgene-containing donor plasmid. Nat. Protoc. 2007, 2, 2325-2331. [CrossRef] [PubMed]

34. Groth, A.C.; Fish, M.; Nusse, R.; Calos, M.P. Construction of transgenic Drosophila by using the site-specific integrase from phage phiC31. Genetics 2004, 166, 1775-1782. [CrossRef] [PubMed] 
35. Tsou, W.L.; Hosking, R.R.; Burr, A.A.; Sutton, J.R.; Ouyang, M.; Du, X.; Gomez, C.M.; Todi, S.V. DnaJ-1 and karyopherin alpha3 suppress degeneration in a new Drosophila model of Spinocerebellar Ataxia Type 6. Hum. Mol. Genet. 2015, 24, 4385-4396. [CrossRef]

36. Tsou, W.L.; Qiblawi, S.H.; Hosking, R.R.; Gomez, C.M.; Todi, S.V. Polyglutamine length-dependent toxicity from alpha1ACT in Drosophila models of spinocerebellar ataxia type 6. Biol. Open 2016, 5, 1770-1775. [CrossRef] [PubMed]

37. Sujkowski, A.; Bazzell, B.; Carpenter, K.; Arking, R.; Wessells, R.J. Endurance exercise and selective breeding for longevity extend Drosophila healthspan by overlapping mechanisms. Aging (Albany NY) 2015, 7, 535-552. [CrossRef]

38. Brand, A.H.; Manoukian, A.S.; Perrimon, N. Ectopic expression in Drosophila. Methods Cell Biol. 1994, 44, 635-654.

39. Brand, A.H.; Perrimon, N. Targeted gene expression as a means of altering cell fates and generating dominant phenotypes. Development (Cambridge, England) 1993, 118, 401-415.

40. Sutton, J.R.; Blount, J.R.; Libohova, K.; Tsou, W.L.; Joshi, G.S.; Paulson, H.L.; Costa, M.D.C.; Scaglione, K.M.; Todi, S.V. Interaction of the polyglutamine protein ataxin-3 with Rad23 regulates toxicity in Drosophila models of Spinocerebellar Ataxia Type 3. Hum. Mol. Genet. 2017, 26, 1419-1431. [CrossRef]

41. Ristic, G.; Sutton, J.R.; Libohova, K.; Todi, S.V. Toxicity and aggregation of the polyglutamine disease protein, ataxin-3 is regulated by its binding to VCP/p97 in Drosophila melanogaster. Neurobiol. Dis. 2018, 116, 78-92. [CrossRef]

42. Johnson, S.L.; Blount, J.R.; Libohova, K.; Ranxhi, B.; Paulson, H.L.; Tsou, W.L.; Todi, S.V. Differential toxicity of ataxin-3 isoforms in Drosophila models of Spinocerebellar Ataxia Type 3. Neurobiol. Dis. 2019, 132, 104535. [CrossRef] [PubMed]

43. Weber, A.; Elliott, P.R.; Pinto-Fernandez, A.; Bonham, S.; Kessler, B.M.; Komander, D.; El Oualid, F.; Krappmann, D. A Linear Diubiquitin-Based Probe for Efficient and Selective Detection of the Deubiquitinating Enzyme OTULIN. Cell Chem. Biol. 2017, 24, 1299-1313.e1297. [CrossRef] [PubMed]

44. Todi, S.V.; Franke, J.D.; Kiehart, D.P.; Eberl, D.F. Myosin VIIA defects, which underlie the Usher 1B syndrome in humans, lead to deafness in Drosophila. Curr. Biol. 2005, 15, 862-868. [CrossRef] [PubMed]

45. Franke, J.D.; Dong, F.; Rickoll, W.L.; Kelley, M.J.; Kiehart, D.P. Rod mutations associated with MYH9-related disorders disrupt nonmuscle myosin-IIA assembly. Blood 2005, 105, 161-169. [CrossRef]

46. Chau, V.; Tobias, J.W.; Bachmair, A.; Marriott, D.; Ecker, D.J.; Gonda, D.K.; Varshavsky, A. A multiubiquitin chain is confined to specific lysine in a targeted short-lived protein. Science 1989, 243, 1576-1583. [CrossRef]

47. Jin, L.; Williamson, A.; Banerjee, S.; Philipp, I.; Rape, M. Mechanism of ubiquitin-chain formation by the human anaphase-promoting complex. Cell 2008, 133, 653-665. [CrossRef]

48. Chen, J.; Chen, Z.J. Regulation of NF-kappaB by ubiquitination. Curr. Opin. Immunol. 2013, 25, 4-12. [CrossRef]

49. Courtois, G.; Fauvarque, M.O. The Many Roles of Ubiquitin in NF-kappaB Signaling. Biomedicines 2018, 6, 43. [CrossRef]

50. Xia, Z.P.; Sun, L.; Chen, X.; Pineda, G.; Jiang, X.; Adhikari, A.; Zeng, W.; Chen, Z.J. Direct activation of protein kinases by unanchored polyubiquitin chains. Nature 2009, 461, 114-119. [CrossRef]

51. Skaug, B.; Chen, J.; Du, F.; He, J.; Ma, A.; Chen, Z.J. Direct, noncatalytic mechanism of IKK inhibition by A20. Mol. Cell. 2011, 44, 559-571. [CrossRef] [PubMed]

52. Zeng, W.; Sun, L.; Jiang, X.; Chen, X.; Hou, F.; Adhikari, A.; Xu, M.; Chen, Z.J. Reconstitution of the RIG-I pathway reveals a signaling role of unanchored polyubiquitin chains in innate immunity. Cell 2010, 141, 315-330. [CrossRef] [PubMed]

53. Catici, D.A.; Horne, J.E.; Cooper, G.E.; Pudney, C.R. Polyubiquitin Drives the Molecular Interactions of the NF-kappaB Essential Modulator (NEMO) by Allosteric Regulation. J. Biol. Chem. 2015, 290, 14130-14139. [CrossRef] [PubMed]

54. Rahighi, S.; Ikeda, F.; Kawasaki, M.; Akutsu, M.; Suzuki, N.; Kato, R.; Kensche, T.; Uejima, T.; Bloor, S.; Komander, D.; et al. Specific recognition of linear ubiquitin chains by NEMO is important for NF-kappaB activation. Cell 2009, 136, 1098-1109. [CrossRef] [PubMed]

55. Dittmar, G.; Winklhofer, K.F. Linear Ubiquitin Chains: Cellular Functions and Strategies for Detection and Quantification. Front. Chem. 2019, 7, 915. [CrossRef] 
56. Hicke, L.; Schubert, H.L.; Hill, C.P. Ubiquitin-binding domains. Nat. Rev. Mol. Cell Biol. 2005, 6, 610-621. [CrossRef]

57. Laplantine, E.; Fontan, E.; Chiaravalli, J.; Lopez, T.; Lakisic, G.; Veron, M.; Agou, F.; Israel, A. NEMO specifically recognizes K63-linked poly-ubiquitin chains through a new bipartite ubiquitin-binding domain. EMBO J. 2009, 28, 2885-2895. [CrossRef]

58. Sato, Y.; Yoshikawa, A.; Yamashita, M.; Yamagata, A.; Fukai, S. Structural basis for specific recognition of Lys 63-linked polyubiquitin chains by NZF domains of TAB2 and TAB3. EMBO J. 2009, 28, 3903-3909. [CrossRef]

59. Sasaki, C.Y.; Barberi, T.J.; Ghosh, P.; Longo, D.L. Phosphorylation of RelA/p65 on serine 536 defines an I\{kappa\}B\{alpha\}-independent NF-\{kappa\}B pathway. J. Biol. Chem. 2005, 280, 34538-34547. [CrossRef]

60. Choe, K.M.; Werner, T.; Stoven, S.; Hultmark, D.; Anderson, K.V. Requirement for a peptidoglycan recognition protein (PGRP) in Relish activation and antibacterial immune responses in Drosophila. Science 2002, 296, 359-362. [CrossRef]

61. Gottar, M.; Gobert, V.; Michel, T.; Belvin, M.; Duyk, G.; Hoffmann, J.A.; Ferrandon, D.; Royet, J. The Drosophila immune response against Gram-negative bacteria is mediated by a peptidoglycan recognition protein. Nature 2002, 416, 640-644. [CrossRef] [PubMed]

62. Ramet, M.; Manfruelli, P.; Pearson, A.; Mathey-Prevot, B.; Ezekowitz, R.A. Functional genomic analysis of phagocytosis and identification of a Drosophila receptor for E. coli. Nature 2002, 416, 644-648. [CrossRef] [PubMed]

63. Lu, Y.; Wu, L.P.; Anderson, K.V. The antibacterial arm of the drosophila innate immune response requires an IkappaB kinase. Genes Dev. 2001, 15, 104-110. [CrossRef] [PubMed]

64. Rutschmann, S.; Jung, A.C.; Zhou, R.; Silverman, N.; Hoffmann, J.A.; Ferrandon, D. Role of Drosophila IKK gamma in a toll-independent antibacterial immune response. Nat. Immunol. 2000, 1, 342-347. [CrossRef] [PubMed]

65. Silverman, N.; Zhou, R.; Stoven, S.; Pandey, N.; Hultmark, D.; Maniatis, T. A Drosophila IkappaB kinase complex required for Relish cleavage and antibacterial immunity. Genes Dev. 2000, 14, 2461-2471. [CrossRef] [PubMed]

66. Stoven, S.; Ando, I.; Kadalayil, L.; Engstrom, Y.; Hultmark, D. Activation of the Drosophila NF-kappaB factor Relish by rapid endoproteolytic cleavage. EMBO Rep. 2000, 1, 347-352. [CrossRef]

67. Stoven, S.; Silverman, N.; Junell, A.; Hedengren-Olcott, M.; Erturk, D.; Engstrom, Y.; Maniatis, T.; Hultmark, D. Caspase-mediated processing of the Drosophila NF-kappaB factor Relish. Proc. Natl. Acad. Sci. USA 2003, 100, 5991-5996. [CrossRef]

68. Myllymaki, H.; Valanne, S.; Ramet, M. The Drosophila imd signaling pathway. J. Immunol. 2014, 192, 3455-3462. [CrossRef]

69. Chen, Z.; Pickart, C.M. A 25-kilodalton ubiquitin carrier protein (E2) catalyzes multi-ubiquitin chain synthesis via lysine 48 of ubiquitin. J. Biol. Chem. 1990, 265, 21835-21842.

70. Park, E.S.; Elangovan, M.; Kim, Y.J.; Yoo, Y.J. UbcD4, an ortholog of E2-25K/Ube2K, is essential for activation of the immune deficiency pathway in Drosophila. Biochem. Biophys. Res. Commun. 2016, 469, 891-896. [CrossRef]

71. Zhou, R.; Silverman, N.; Hong, M.; Liao, D.S.; Chung, Y.; Chen, Z.J.; Maniatis, T. The role of ubiquitination in Drosophila innate immunity. J. Biol. Chem. 2005, 280, 34048-34055. [CrossRef] [PubMed]

72. Bai, Z.; Li, Z.; Xiao, W. Drosophila bendless catalyzes K63-linked polyubiquitination and is involved in the response to DNA damage. Mutat. Res. 2018, 808, 39-47. [CrossRef] [PubMed]

73. Arnot, C.J.; Gay, N.J.; Gangloff, M. Molecular mechanism that induces activation of Spatzle, the ligand for the Drosophila Toll receptor. J. Biol. Chem. 2010, 285, 19502-19509. [CrossRef] [PubMed]

74. Gangloff, M.; Murali, A.; Xiong, J.; Arnot, C.J.; Weber, A.N.; Sandercock, A.M.; Robinson, C.V.; Sarisky, R.; Holzenburg, A.; Kao, C.; et al. Structural insight into the mechanism of activation of the Toll receptor by the dimeric ligand Spatzle. J. Biol. Chem. 2008, 283, 14629-14635. [CrossRef]

75. Weber, A.N.; Moncrieffe, M.C.; Gangloff, M.; Imler, J.L.; Gay, N.J. Ligand-receptor and receptor-receptor interactions act in concert to activate signaling in the Drosophila toll pathway. J. Biol. Chem. 2005, 280, 22793-22799. [CrossRef]

76. Towb, P.; Bergmann, A.; Wasserman, S.A. The protein kinase Pelle mediates feedback regulation in the Drosophila Toll signaling pathway. Development 2001, 128, 4729-4736. 
77. Wu, L.P.; Anderson, K.V. Regulated nuclear import of Rel proteins in the Drosophila immune response. Nature 1998, 392, 93-97. [CrossRef]

78. Valanne, S.; Wang, J.H.; Ramet, M. The Drosophila Toll signaling pathway. J. Immunol. 2011, 186, 649-656. [CrossRef]

79. Ugur, B.; Chen, K.; Bellen, H.J. Drosophila tools and assays for the study of human diseases. Dis. Model Mech. 2016, 9, 235-244. [CrossRef] 